\title{
Upregulation of the inwardly rectifying potassium channel Kir2.1 (KCNJ2) modulates multidrug resistance of small-cell lung cancer under the regulation of miR-7 and the Ras/MAPK pathway
}

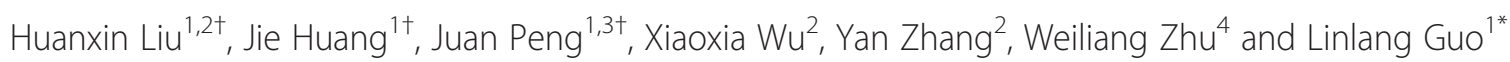

\begin{abstract}
Background: KCNJ2/Kir2.1, a member of the classical inwardly rectifying potassium channel family, is commonly expressed in a wide range of tissues and cell types. Previous studies indicated that Kir2.1 may be associated with SCLC multidrug resistance (MDR). However, whether Kir2.1 can regulate MDR and its underlying mechanisms remain poorly understood in SCLC.

Methods: KCNJ2/Kir2.1 expression was examined in tissues from fifty-two SCLC cases by immunohistochemistry. Overexpression or knockdown of KCNJ2/Kir21 was performed in multidrug-resistant SCLC cell lines (H69AR and H446AR) and their parental cell lines (H69 and H446) to assess its influence on cell growth, apoptosis, the cell cycle and chemoresistance.

Results: KCNJ2/Kir2.1 was expressed in 44.23\% (23/52) of SCLC tissues. Overexpression of KCNJ2/Kir2.1 was correlated with the clinical stage and chemotherapy response in SCLC patients. Knockdown of KCNJ2/Kir2.1 expression using KCNJ2/Kir2.1 shRNA in H69AR and H446AR cells inhibited cell growth and sensitized the cancer cells to chemotherapeutic drugs by increasing cell apoptosis and cell cycle arrest. Forced KCNJ2/Kir2.1 expression in H69 and H446 cells promoted cell growth and enhanced multidrug resistance via reduced drug-induced apoptosis accompanied by cell cycle arrest. KCNJ2/Kir2.1 expression was also influenced by PKC and MEK inhibitors. In addition, multidrug resistance protein 1 (MRP1/ABCC1) was confirmed to interact with KCNJ2/Kir2.1 by Co-IP assays.

Conclusions: KCNJ2/Kir2.1 modulates cell growth and drug resistance by regulating MRP1/ABCC1 expression and is simultaneously regulated by the Ras/MAPK pathway and miR-7. KCNJ2/Kir2.1 may be a prognostic predictor and a potentially novel target for interfering with chemoresistance in SCLC.
\end{abstract}

Keywords: SCLC, KCNJ2/Kir2.1, Chemoresistance, MRP1/ABCC1, miR-7, Ras/MAPK pathway

\section{Background}

Lung cancer, with 1.35 million new cases and causing more than 1 million deaths each year, is the most common cancer and the leading cause of cancer-related deaths worldwide [1]. Small-cell lung cancer (SCLC), the most aggressive type of lung cancer, constitutes approximately $15-18 \%$ of all lung cancers [2]. According to the

\footnotetext{
* Correspondence: linlangg@yahoo.com

${ }^{\dagger}$ Equal contributors

'Department of Pathology, Zhujiang Hospital, Southern Medical University, Guangzhou, China

Full list of author information is available at the end of the article
}

Veterans Administration Lung Group system, SCLC is traditionally defined by a two-stage classification system that includes limited disease and extensive disease. At present, chemotherapy remains the first treatment option for SCLC patients. Although 80-90\% of SCLC patients are initially responsive to chemotherapy, most of them succumb to the disease within a year due to rapidly developing multidrug resistance (MDR) $[3,4]$. Thus, MDR has become the main obstacle to the treatment of SCLC and a central issue in improving its prognosis.

Kir2.1, encoded by the KCNJ2 gene, is a member of the classical inwardly rectifying potassium channel family 
(Kir2 subfamily). It conducts a strong inward rectifier $\mathrm{K}^{+}$ current in a wide range of tissues and cell types, including neurons, skeletal muscle, cardiac myocytes, and immune system and carcinoma cells [5]. The KCNJ2 gene was first cloned by Kubo et al. from a macrophage cell line in 1993 [6]. Similar to the other members of the Kir family, Kir2.1 is tetrameric, containing two transmembrane helix domains (M1 and M2), an ion-selective P-loop between M1 and $\mathrm{M} 2$, and cytoplasmic $\mathrm{N}$ - and C-terminal domains. Functionally, Kir2.1 plays a key role in maintaining the resting membrane potential and regulating cellular excitability in SCLC cells, cardiac myocytes, skeletal muscle and neurons [7-9]. Changes in the expression levels of $\mathrm{K}^{+}$ channels induced by aberrant KCNJ2 expression have substantial effects on cellular processes such as cell death, apoptosis, proliferation and adhesion, which is linked to a variety of cardiac and neurological disorders [10-15]. Human SCLC cells are suggested to be of neurorctodermal origin and exhibit electrophysiological characteristics typical of neuroendocrine cells. Previous studies have indicated that the large, inwardly rectifying $\mathrm{K}+$ current is generated by Kir2.1 and may be associated with SCLC cell MDR [16,17]. However, whether Kir2.1 can regulate MDR and its underlying mechanisms remain poorly understood in SCLC.

MicroRNAs (miRNAs) are a class of small, non-coding RNAs of 18-24 nucleotides in length that negatively regulate the expression of specific genes by binding to the 3' untranslated region (3'UTR) of an mRNA, leading to either translational inhibition or mRNA degradation [18]. Recent evidence has shown that more than $50 \%$ of miRNAs are located in cancer-associated genomic break points and can function as tumor suppressor genes or oncogenes depending on their targets $[19,20]$. Moreover, extensive studies have indicated that miRNAs are closely related to responses to chemotherapeutic treatment [21-24]. For example, Yang et al. reported that miR-214 induced cell survival and cisplatin resistance in ovarian cancer [25]. Additionally, miR-650 levels affected the chemosensitivity of lung adenocarcinoma cells to docetaxel via $\mathrm{Bcl}-2 / \mathrm{Bax}$ expression regulation by directly targeting ING4 [23], and suppression of miR-137 expression in a drug-resistant SCLC cell line increased its sensitivity to cisplatin [26]. Moreover, our previous miRNA expression profile study revealed that the expression of $61 / 852$ miRNAs was significantly increased (>3-fold) in MDR SCLC H69AR cells compared with their drug-sensitive parental cell line H69, suggesting a role for these differentially expressed miRNAs in the development of drug resistance in SCLC cells [22].

We previously found that KCNJ2 is overexpressed in H69AR cells compared to parental H69 cells, whereas miR-7 is expressed at a lower level in H69AR cells compared with $\mathrm{H} 69$ cells (unpublished data). In the present study, we further investigated the roles of KCNJ2/Kir2.1 in drug resistance using human drug-resistant SCLC cell lines (H69AR and H446AR). The correlation between $\mathrm{KCNJ} 2$ expression and clinical drug response was analyzed in SCLC patients. We then validated the interaction between Kir2.1 and MRP1/ABCC1 by coimmunoprecipitation (Co-IP). Furthermore, we showed that KCNJ2 was modulated by the Ras/MAPK pathway and directly targeted by miR-7. Collectively, our results provide a novel explanation for the chemoresistance of SCLC and suggest that KCNJ2/Kir2.1 plays a crucial role in SCLC MDR.

\section{Results}

Kir2.1 expression is associated with the clinical stage and the chemotherapy Response of SCLC patients

To investigate the clinical features of Kir2.1 expression in SCLC, we first examined the expression levels of Kir2.1 in 52 SCLC specimens and 15 normal lung tissues by immunohistochemistry (IHC). Kir2.1 was localized on the membrane of the cancer cells (Figure 1A), whereas no positive Kir2.1 staining was observed in normal lung alveolar epithelium (Figure 1B). And the corresponding IHC staining scores are indicated in Additional file 1A: Figure S1A. Additionally, the positive expression of Kir2.1 was 23 of $52(44 \%)$ in SCLC and 1 of 15 in normal bronchial epithelium, respectively (Figure 1E).

We further analyzed the correlation between Kir2.1 expression and the clinicopathological features of SCLC patients. As shown in Table 1, Kir2.1 expression was significantly more frequent at the extensive disease stage and in the drug-resistant group than at the limited disease stage and in the drug-sensitive group. Correlation analysis showed that Kir2.1 expression was significantly associated with clinical stage and chemotherapy response in SCLC patients $(P<0.05)$, but not with gender, age or smoking history $(P>0.05)$ (Table 1$)$.

\section{KCNJ2/Kir2.1 expression is correlated with}

\section{chemoresistance in SCLC}

Our previous study indicated a 4.75 -fold upregulation of KCNJ2 expression in H69AR cells compared with their parental $\mathrm{H} 69$ cells by cDNA microarray analysis (unpublished data). We confirmed the result in two pairs of SCLC cells (H69AR/H69 and H446AR/H446) by qRTPCR, western blotting and immunofluorescence assays. Figure 2 shows that KCNJ2/Kir2.1 expression was significantly increased at both mRNA and protein levels in H69AR and H446AR cells as compared with H69 and H446 cells, respectively.

Based on the upregulation of KCNJ2/Kir2.1 in MDR SCLC cells, we hypothesized that KCNJ2/Kir2.1 may be involved in SCLC chemoresistance. To test this hypothesis, we performed gain-of-function and loss-of-function 

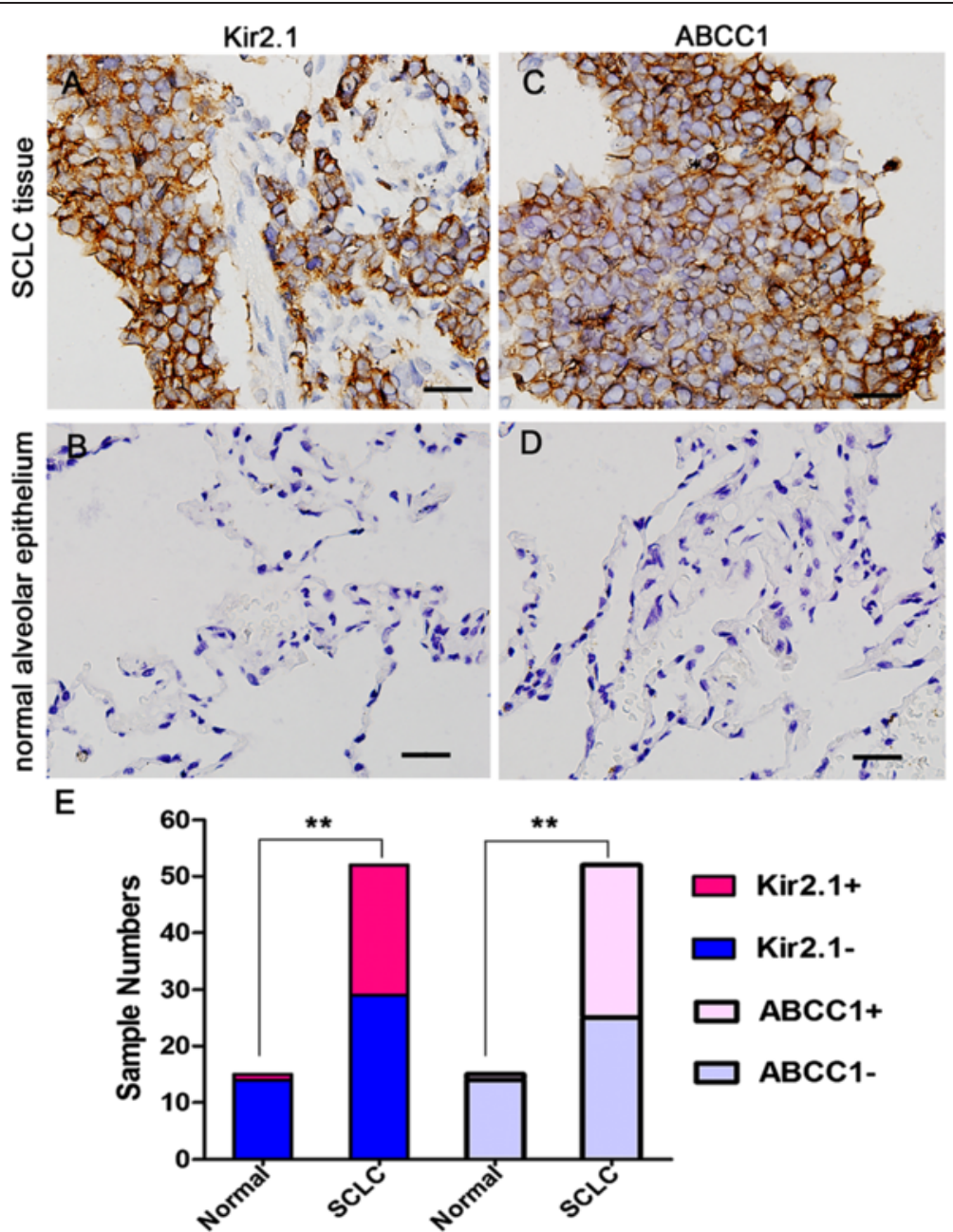

Figure 1 Kir2.1 and MRP1/ABCC1 expression in SCLC and normal alveolar epithelium tissues by IHC. (A-B) Representative images of Kir2.1 IHC staining in SCLC (A) and normal alveolar epithelium tissues (B). (C-D) Representative images of MRP1/ABCC1 IHC staining in SCLC (C) and normal alveolar epithelium tissues (D). Brown chromogen represents positive staining. Scale bar, $50 \mu \mathrm{m}$. (E) The IHC staining results of Kir2.1 and MRP1/ABCC1 expression in normal alveolar epithelium and SCLC tissues. The positive rate of Kir2.1 and MRP1/ABCC1 were higher in SCLC tissues than normal alveolar epithelium. ${ }^{* *}, P<0.01$ compared with normal alveolar epithelium.

studies. First, we developed two stable KCNJ2/Kir2.1overexpressing sublines, H69-KCNJ2 and H446-KCNJ2, by transfecting $\mathrm{H} 69$ and $\mathrm{H} 446$ cells with pcDNA3.1$\mathrm{KCNJ} 2$, and their corresponding negative control sublines (H46-NC and H446-NC) that were transfected with pcDNA3.1 empty vector. qRT-PCR and western blotting analyses showed that KCNJ2/Kir2.1 expression was significantly upregulated at both the mRNA and protein levels in H69-KCNJ2 and H446-KCNJ2 as compared with H46-NC and H446-NC, respectively (Figure 3). Subsequently, we stably transfected multidrug-resistant H69AR and H446AR cells with two shRNAs (shKCNJ2-1 and shKCNJ2-2) targeting KCNJ2 to inhibit KCNJ2/Kir2.1 expression. As shown in Figure 3, KCNJ2/Kir2.1 expression was notably decreased in cells transfected with
shKCNJ2-1 or shKCNJ2-2 (H69AR-shKCNJ2-1, H69ARshKCNJ2-2, H446AR-shKCNJ2-1 and H446AR-shKCNJ22) when compared with their corresponding negative controls, H69AR-shNC or H446AR-shNC. The viability and sensitivity of the SCLC cells to chemotherapeutic drugs (ADM, CDDP and VP-16) were then assessed using CCK8 assays. Table 2 shows that the $\mathrm{IC}_{50}$ values of $\mathrm{H} 69$ and $\mathrm{H} 446$ cells significantly increased after transfection with pcDNA3.1-KCNJ2, whereas the $\mathrm{IC}_{50}$ values of H69AR and H446AR cells decreased after the introduction of shKCNJ2-1 or shKCNJ2-2. Collectively, these results indicated that $\mathrm{KCNJ} 2 / \mathrm{Kir} 2.1$ upregulation or downregulation could significantly affect the sensitivity of SCLC cells to chemotherapeutic drugs, suggesting that KCNJ2/Kir2.1 expression may be associated with chemoresistance in SCLC. 
Table 1 The expression of Kir2.1 and MRP1/ABCC1 and their relationships with the clinicopathological characteristics in SCLC patients

\begin{tabular}{|c|c|c|c|c|c|c|c|}
\hline \multirow{2}{*}{$\begin{array}{l}\text { Clinic pathological } \\
\text { features }\end{array}$} & \multirow[t]{2}{*}{$\mathbf{n}$} & \multicolumn{3}{|c|}{ Expression of Kir2.1 } & \multicolumn{3}{|c|}{ Expression of MRP1/ABCC1 } \\
\hline & & + & - & $P$ & + & - & $P$ \\
\hline Gender & & & & 1 & & & 1 \\
\hline Male & 43 & 18 & 25 & & 21 & 22 & \\
\hline Female & 9 & 5 & 4 & & 6 & 3 & \\
\hline Age & & & & 0.892 & & & 0.426 \\
\hline$<55^{\dagger}$ & 23 & 7 & 16 & & 10 & 13 & \\
\hline$\geq 55$ & 29 & 16 & 13 & & 17 & 12 & \\
\hline Smoking history & & & & 1 & & & 0.328 \\
\hline Yes & 41 & 20 & 21 & & 22 & 19 & \\
\hline No & 11 & 3 & 8 & & 5 & 6 & \\
\hline Clinical stage & & & & 0.032 & & & 0.001 \\
\hline limited disease & 25 & 7 & 18 & & 6 & 19 & \\
\hline extensive disease & 27 & 16 & 11 & & 21 & 6 & \\
\hline Chemotherapy reponse & & & & 0.021 & & & 0.001 \\
\hline Sensitive group & 25 & 6 & 19 & & 7 & 18 & \\
\hline Resistant group & 22 & 17 & 5 & & 20 & 2 & \\
\hline
\end{tabular}

${ }^{\dagger}$ median age.

-, negative expression; +, positive expression.

The P-value was calculated by $x^{2}$ test.

KCNJ2/Kir2.1 induces cell cycle arrest and apoptosis following exposure to chemotherapeutic drugs

The above observations prompted us to investigate the possible mechanisms of KCNJ2/Kir2.1 in SCLC chemoresistance. Using flow cytometry analysis, we evaluated the effect of KCNJ2/Kir2.1 on cell cycle control and apoptosis following exposure to chemotherapeutic drugs.

As shown in Figure 4, overexpression of $\mathrm{KCNJ} 2$ / Kir2.1 in H69 and H446 cells induced an increase of cells entering the G0/G1 phase and a corresponding decline of cells in the $S$ phase after treatment with ADM $(4.6 \mu \mathrm{M})$ for $24 \mathrm{~h}$. In contrast, knockdown of KCNJ2/ Kir2.1 by shKCNJ2-1 or shKCNJ2-2 in H69AR and H446AR cells resulted in a significant decrease of cells in the G0/G1 phase and an increase of cells in the $\mathrm{S}$ phase. These results suggest that $\mathrm{KCNJ} 2 / \mathrm{Kir} 2.1$ could induce cell cycle arrest.

We further examined the impact of $\mathrm{KCNJ} 2 / \mathrm{Kir} 2.1$ on apoptosis. Notably, after treatment with ADM $(4.6 \mu \mathrm{M})$ for $24 \mathrm{~h}$, upregulating $\mathrm{KCNJ} 2 / \mathrm{Kir} 2.1$ expression in $\mathrm{H} 69$ and $\mathrm{H} 446$ cells prevented drug-induced apoptosis. Meanwhile, silencing KCNJ2/Kir2.1 expression in H69AR and H446AR cells significantly increased drug-induced apoptosis (Figure 5). In addition, similar results of cell cycle and apoptosis analysis were observed in SCLC cells after treatment with CDDP $(16.7 \mu \mathrm{M})$ or VP-16 $(34 \mu \mathrm{M})$ (Additional file 2: Figure S2, Additional file 3: Figure S3, Additional file 4: Figure S4, and Additional file 5: Figure S5).

\section{KCNJ2/Kir2.1 enhances SCLC tumor growth in vivo}

To further determine the effect of $\mathrm{KCNJ} 2 / \mathrm{Kir} 2.1$ on tumor growth in vivo, SCLC cells with altered KCNJ2/ Kir2.1 expression were subcutaneously injected into nude mice. As shown in Figure 6A-D, the injection of KCNJ2/Kir2.1-overexpressing H69 and H446 cells resulted in significantly increased tumor weights compared with the injection of the corresponding NC cells. However, with KCNJ2/Kir2.1-knockdown H69AR and H446AR cells, the average tumor weight decreased to $12-25 \%$ of that of the tumors originating from the cells transfected with shNC. Additionally, the injection of KCNJ2/Kir2.1-overexpressing H69 and H446 cells resulted in earlier tumor formation compared with the injection of NC cells. In contrast, the time until tumor appearance was prolonged in H69AR and H446AR cells transfected with shKCNJ2-1 or shKCNJ2-2 compared with that transfected with shNC. These findings demonstrated that KCNJ2/Kir2.1 enhanced SCLC tumor growth in vivo.

We then further examined Kir2.1 expression in SCLC tissues from the tumor xenografts by IHC. The results showed higher Kir2.1 expression in H69-KCNJ2 tumors and lower expression in shKCNJ2-1 H69AR tumors as compared with the corresponding NC cells (Figure 6E and Additional file 1B: Figure S1B). Moreover, evaluated by staining scores, Kir2.1 and MRP1/ABCC1 expression were significantly upregulated in tumors originating from $\mathrm{H} 69$ and $\mathrm{H} 446$ cells transfected with KCNJ2overexpressing vector and downregulated in tumors of 
A

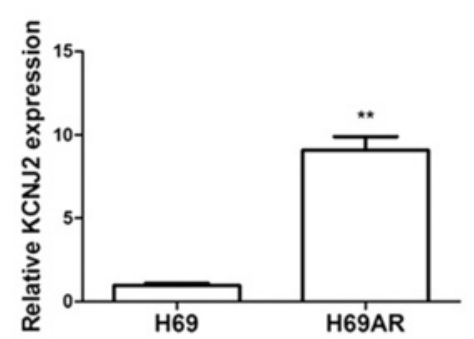

B
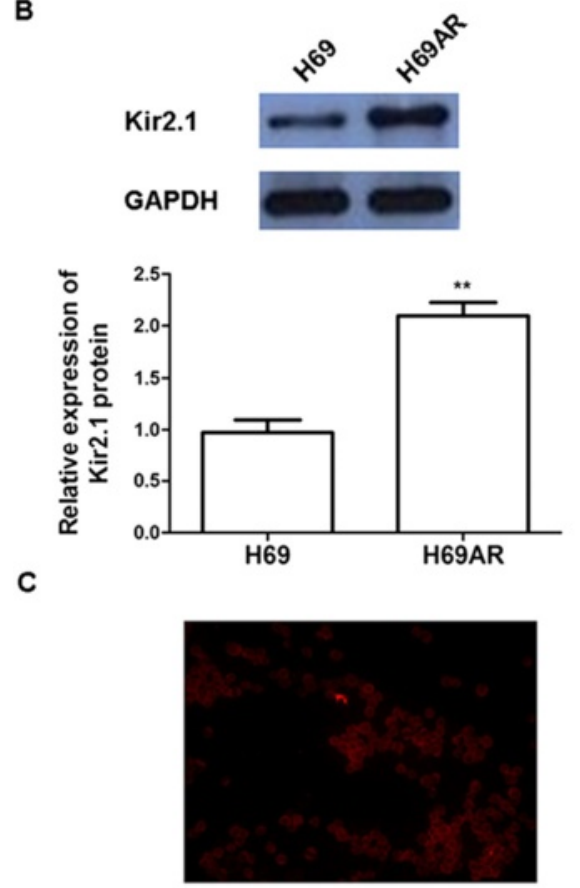

H69

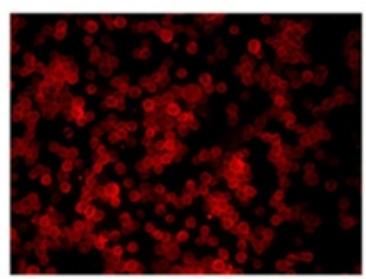

H69AR

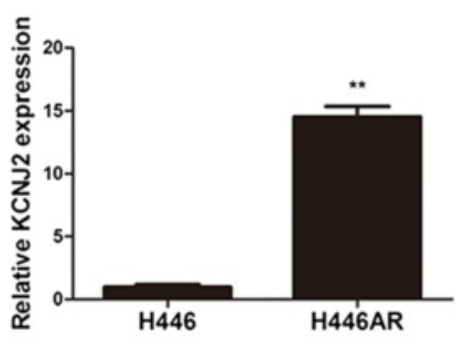

Kir2.1

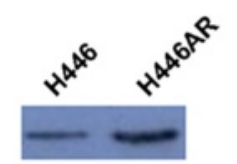

GAPDH
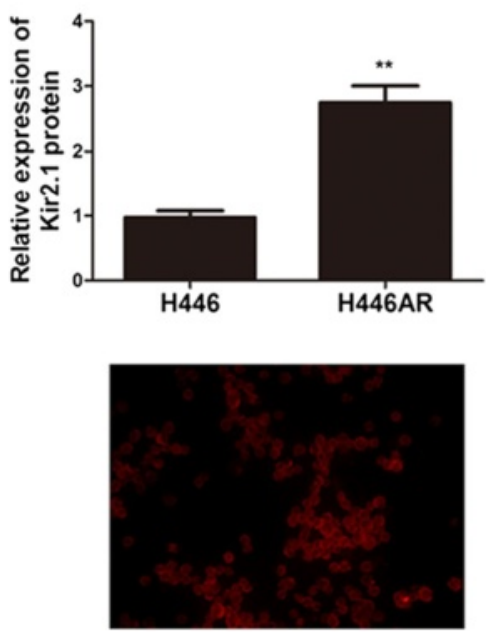

H446

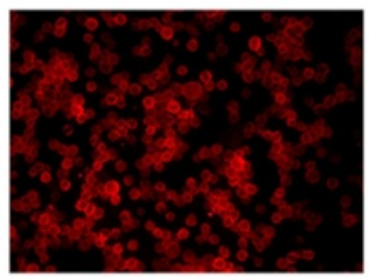

H446AR

Figure 2 KCNJ2/Kir2.1 expressionin SCLC cells. (A) KCNJ2 mRNA level was determined by qRT-PCR in SCLC multi-drug resistant H69AR and H446AR cells and their corresponding parental cells, H69 and H446. (B-C) Western blotting and immunofluorescence analyses showed that Kir2.1 expression was upregulatedin H69ARand H44AR cellsas compared with H69 and H446 cells, respectively. Values of three independent experiments are represented as the mean $\pm \mathrm{SD} .{ }^{* *}, P<0.01$ compared with the parental cells.

H69AR and H446AR cells transfected with shKCNJ2-1 or shKCNJ2-2 (Figure 6F).

\section{KCNJ2/Kir2.1 interacts with MRP1/ABCC1}

We previously found that the expression of both $\mathrm{KCNJ} 2 /$ Kir2.1 and MRP1/ABCC1 was increased in H69AR cells compared with parental H69 cells by cDNA microarray analysis [22]. To further investigate the relationship between KCNJ2/Kir2.1 and MRP1/ABCC1 in SCLC, we simultaneously detected the expression of these two proteins in SCLC and normal lung tissues by IHC. Membrane localization of KCNJ2/Kir2.1 and MRP1/ABCC1 was observed in cancer cells (Figure $1 \mathrm{~A}$ and $\mathrm{C}$ ), whereas no positive MRP1/ABCC1 staining was observed in normal lung alveolar epithelium (Figure 1D). Similar to Kir2.1, the rate of MRP1/ABCC1 positivity was much higher in SCLC specimens (52\%) than in normal bronchial epithelium (7\%) (Figure 1E). Correlation analysis showed that MRP1/ 
A
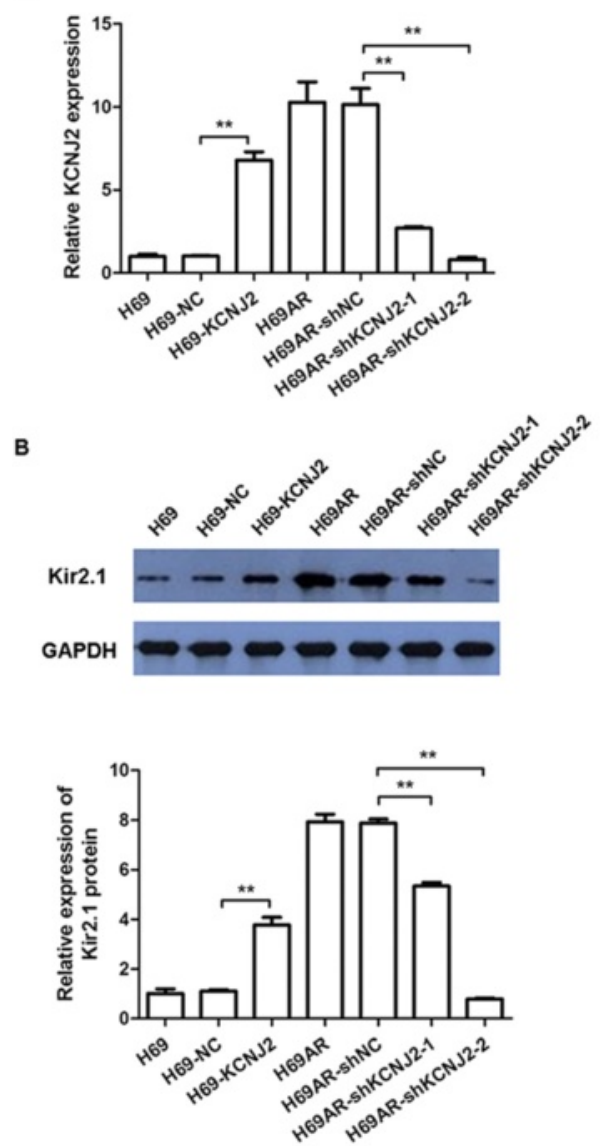
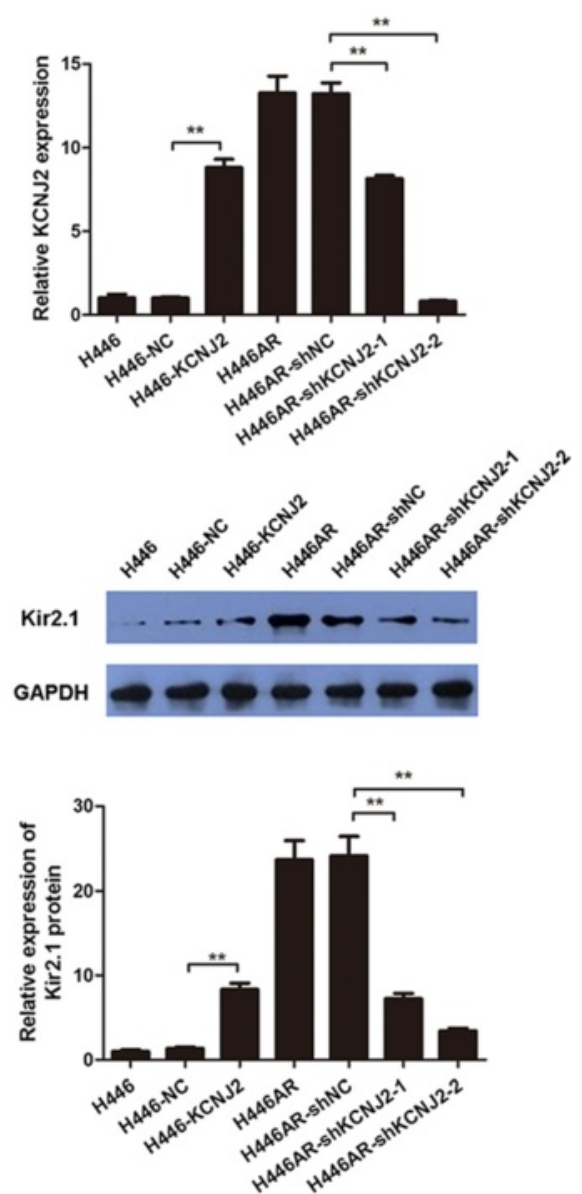

Figure 3 Altered KCNJ2/Kir2.1 expression in SCLC cells. qRT-PCR (A) and Western blot (B) analyses showed forced KCNJ2/Kir2.1 expression in H69 and H446 cells after transfection of pcDNA3.1-KCNJ2, and KCNJ2/Kir2.1 inhibition in H69AR and H446AR cells by shKCNJ2-10r shKCNJ2-2. The bars represent the means \pm SD of three independent experiments. ${ }^{*}, P<0.01$ compared withthe corresponding NC cells transfected with pCDNA3.1 empty vector or shNC.

ABCC1 expression was significantly associated with the SCLC patient's clinical stage and chemotherapy response, but not with gender, smoking, age or lymph node metastasis (Table 1). Furthermore, as shown in Table 3, MRP1/ ABCC1 expression was clearly correlated with Kir2.1 expression in SCLC tissues.

To explore the relationship between KCNJ2/Kir2.1 and MRP1/ABCC1, we analyzed MRP1/ABCC1 expression changes with upregulated or downregulated KCNJ2/Kir2.1 expression. qRT-PCR and western blotting analyses revealed a marked increase in MRP1/ABCC1 in H69 and H446 cells transfected with pcDNA3.1-KCNJ2 and a decrease in MRP1/ABCC1in H69AR and H446AR cells transfected with shKCNJ2-1 or shKCNJ2-2 (Figure 7A-B). These results provide evidence indicating that $\mathrm{KCNJ} 2$ / Kir2.1 could affect MRP1/ABCC1 expression.

We performed Co-IP assays to further evaluate the interaction between KCNJ2/Kir2.1 and MRP1/ABCC1 in SCLC cells. Cell lysates from H69 cells were prepared and immunoprecipitated with an anti-Kir2.1 antibody. Both immunoprecipitates and cell lysates without IP were blotted with anti-ABCC1 and antiKir2.1. As shown in Figure 7C, Kir2.1 formed a complex with ABCC1. These data reveal that Kir2.1 can interact with MRP1/ABCC1.

\section{The modulatory effect of the Ras/MAPK pathway on KCNJ2/Kir2.1}

Based on previous findings that the Ras-MAPK pathway is involved in modulating the inward rectifier potassium channel IRK1 [5], we investigated whether the RasMAPK pathway could regulate KCNJ2/Kir2.1 in SCLC. Initially, SCLC MDR H69AR and H446AR cells were treated with PKC inhibitor (staurosporine, $10 \mathrm{nM}$ ) and MEK inhibitor (U0126, $10 \mu \mathrm{M}$ ) to inhibit two key components of Ras-MAPK pathway respectively. Then, we examined the mRNA and protein levels of both $\mathrm{KCNJ} 2 /$ Kir2.1 and MRP1/ABCC1 in these SCLC cells by qRTPCR and Western blotting. The results showed that the expression of both $\mathrm{KCNJ} 2 / \mathrm{Kir} 2.1$ and MRP1/ABCC1 
Table 2 Effect of KCNJ2/Kir2.1 on drug sensitivity $(\bar{X} \pm s, n=3, \mu \mathrm{g} / \mathrm{ml})$

\begin{tabular}{llll}
\hline Cells & IC50 & & \\
\cline { 2 - 4 } & ADM & CDDP & VP-16 \\
\hline H69 & $5.222 \pm 0.117$ & $17.711 \pm 0.279$ & $124.03 \pm 2.791$ \\
H69-NC & $5.426 \pm 0.87$ & $17.548 \pm 0.423$ & $122.27 \pm 2.938$ \\
H69-KCNJ2 & $13.678 \pm 0.195^{*}$ & $22.649 \pm 7.623^{*}$ & $283.41 \pm 6.884^{*}$ \\
H446 & $4.293 \pm 0.049$ & $4.529 \pm 0.063$ & $52.342 \pm 3.0$ \\
H446-NC & $4.412 \pm 0.212$ & $4.884 \pm 0.068$ & $50.296 \pm 1.53$ \\
H446-KCNJ2 & $14.048 \pm 0.242^{*}$ & $15.303 \pm 0.712^{*}$ & $80.064 \pm 4.97^{*}$ \\
H69AR & $211.06 \pm 6.665$ & $76.652 \pm 1.238$ & $531.58 \pm 6.06$ \\
H69AR-shNC & $209.69 \pm 3.157$ & $77.32 \pm 1.362$ & $529.72 \pm 4.704$ \\
H69AR-shKCNJ2-1 & $62.275 \pm 1.214^{*}$ & $44.583 \pm 1.514^{*}$ & $394.74 \pm 19.996^{*}$ \\
H69AR-shKCNJ2-2 & $18.823 \pm 0.935^{*}$ & $33.183 \pm 2.637^{*}$ & $359.51 \pm 8.62^{*}$ \\
H446AR & $39.29 \pm 0.738$ & $133.73 \pm 4.875$ & $179.63 \pm 2.978$ \\
H446AR-shNC & $42.35 \pm 1.388$ & $132.12 \pm 4.434$ & $175.64 \pm 8.726$ \\
H446AR-shKCNJ2-1 & $27.082 \pm 2.289^{*}$ & $72.724 \pm 0.896^{*}$ & $118.31 \pm 4.934^{*}$ \\
H446AR-shKCNJ2-2 & $19.920 \pm 0.688^{*}$ & $21.517 \pm 2.693^{*}$ & $108.51 \pm 5.772^{*}$ \\
\hline
\end{tabular}

${ }^{*} P<0.05$ (compared with corresponding negative control groups).

was significantly downregulated following treatment with staurosporine and U0126 as compared with the controls (Figure 8). Moreover, an additive inhibitory effect on KCNJ2/Kir2.1 and MRP1/ABCC1 expression was observed in SCLC cells upon simultaneous PCK and MEK suppression (Figure 8). These findings suggest that $\mathrm{KCNJ} /$ Kir2.1 modulation is mediated by the Ras-PKCMAPK pathway.

\section{MiR-7 directly regulates KCNJ2 expression in SCLC}

All processes involved in cancer, including apoptosis and proliferation, have been shown to be regulated by small regulatory non-coding RNAs, i.e., miRNAs [27]. Therefore, the above results showing that KCNJ2/Kir2.1 is overexpressed in SCLC led us to hypothesize that KCNJ2/Kir2.1 expression may be regulated by endogenous miRNAs. We utilized three prediction algorithms (PicTar, TarScan and miRBase database) to predict the miRNAs that possibly target KCNJ2 in SCLC. The results showed that miR-7, miR-212 and miR-1 have potential interaction sites in the 3'-untranslated region (3'UTR) of KCNJ2 mRNA. Based on the involvement of miR-7 in drug resistance, we investigated whether KCNJ2 is regulated by miR-7. We transfected an miR-7 agomir or antagomir into SCLC cells to increase or decrease miR-7 expression, respectively (Figure 9A). The levels of KCNJ2/Kir2.1 and MRP1/ABCC1 were then measured by qRT-PCR and Western blotting at $48 \mathrm{~h}$ post-transfection. The expression of both $\mathrm{KCNJ} 2 / \mathrm{Kir} 2.1$ and MRP1/ABCC1 decreased significantly after transfection of miR-7 agomir compared with NC agomir, whereas the miR-7 antagomir clearly upregulated the levels of KCNJ2/Kir2.1 and MRP1/ABCC1 when compared with $\mathrm{NC}$ antagomir (Figure 9B-D).

To test whether miR-7 can regulate $\mathrm{KCNJ} 2 / \mathrm{Kir} 2.1$ via direct interaction with the KCNJ2 3'UTR, we constructed luciferase reporter vectors containing wildtype (psiCHECK2-KCNJ2-wt) or mutated (psiCHECK2KCNJ2-mt) 3'UTRs of KCNJ2mRNA. H69 cells were cotransfected with each vector and either the miR-7 agomir or antagomir or the corresponding $\mathrm{NC}$; the KCNJ2 luciferase activity was measured after cotransfection. As shown in Figure 9E, the miR-7 agomir suppressed luciferase activity when cotransfected with the wildtype reporter vector as compared with $\mathrm{NC}$ agomir, whereas the miR-7 antagomir increased luciferase activity compared with $\mathrm{NC}$ antagomir. In contrast, the luciferase activity of the mutated reporter vector was not affected by simultaneous transfection with the miR-7 agomir or antagomir (Figure 9E). Taken together, these findings suggest that $\mathrm{KCNJ} 2$ is a direct target of miR-7 in SCLC.

\section{Regulation of chemoresistance by KCNJ2/Kir2.1 is partly mediated by miR-7}

To further investigate the effect of miR-7 on the chemoresistance of SCLC cells, we analyzed the sensitivity of SCLC cells to chemotherapeutic drugs (ADM, CDDP and VP-16) after transfection with the miR-7 agomir, antagomir or the corresponding NC. The $\mathrm{IC}_{50}$ values of the chemotherapeutic drugs were significantly decreased after miR-7 agomir transfection compared with the controls. Meanwhile, the $\mathrm{IC}_{50}$ values increased when expression of miR-7 was suppressed by miR-7 antagomir (Figure 10). These results demonstrated that the upregulation of miR-7 expression sensitized the SCLC cells to all three chemotherapeutic drugs, whereas the downregulation of miR-7 desensitized the SCLC cells. Together, these data suggest that miR-7 deregulation may be responsible for the effects of $\mathrm{KCNJ} 2$ on SCLC chemoresistance.

\section{miR-7 expression is associated with Kir2.1 levels and prognosis in SCLC patients}

To further confirm the association between miR-7 and KCNJ2/Kir2.1 expression, we analyzed miR-7 expression in the above-mentioned 52 SCLC tissue specimens by qRT-PCR. The median value of miR-7 expression was chosen as the cut-off point for separating SCLC tissues with low-level miR-7 expression $(n=26)$ from those with high-level miR-7 expression $(n=26)$. Correlation analysis showed that miR-7 expression was inversely correlated with $\mathrm{KCNJ} 2 /$ kir2.1 $(P<0.001)$ and MRP1/ABCC1 expression $(P=0.002)$ (Table 4). Moreover, low-level 


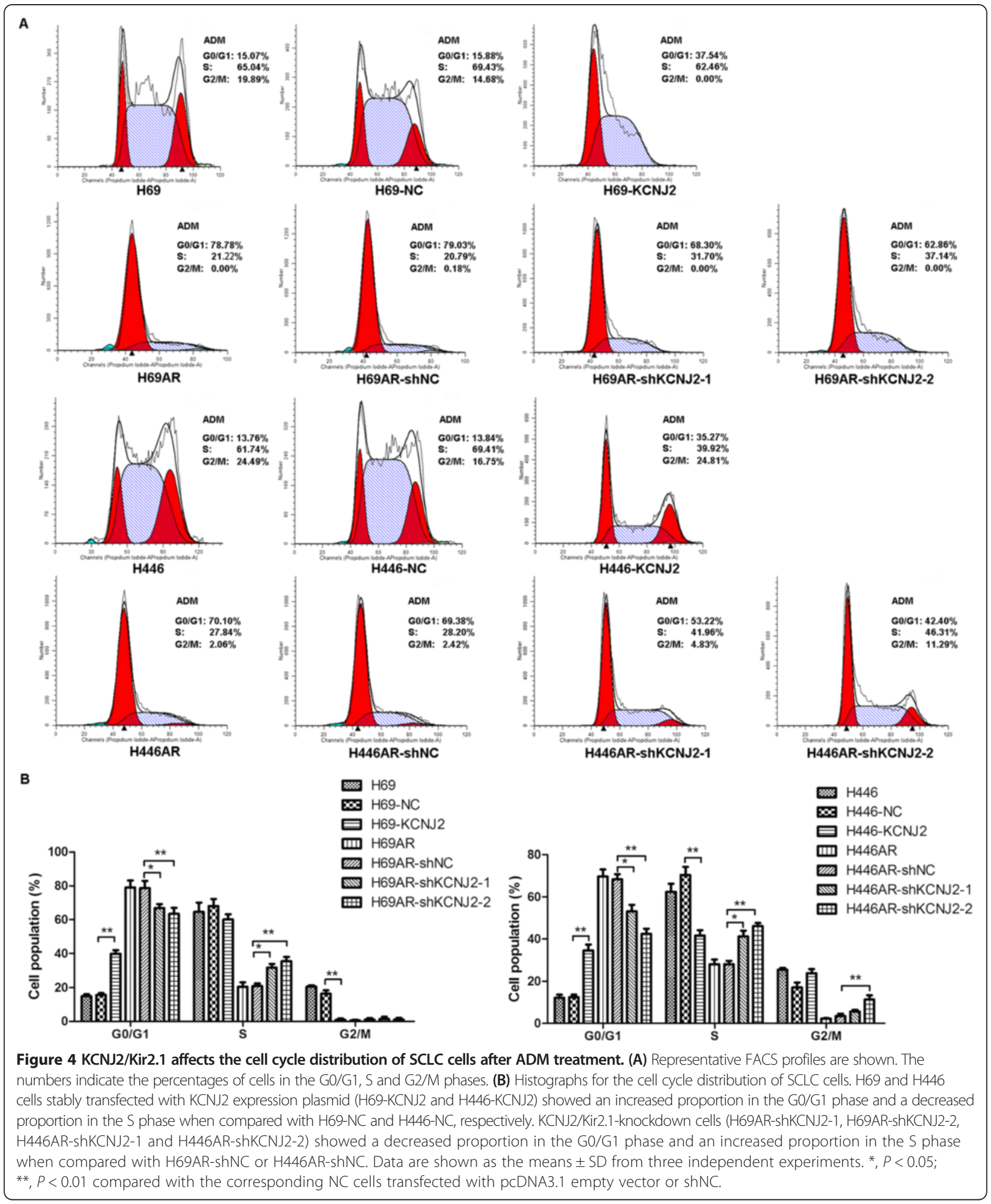

miR-7 expression was significantly associated with a more aggressive clinical stage $(P=0.012)$ (Table 4$)$. KaplanMeier analysis revealed that SCLC patients with low miR7 expression had much shorter overall survival times than patients with high miR-7 expression (Additional file 6: Figure S6). In addition, miR-7 expression reached significance in a univariate Cox proportional hazards regression model ( $P=0.024$, Additional file 7: Table S1). 
A
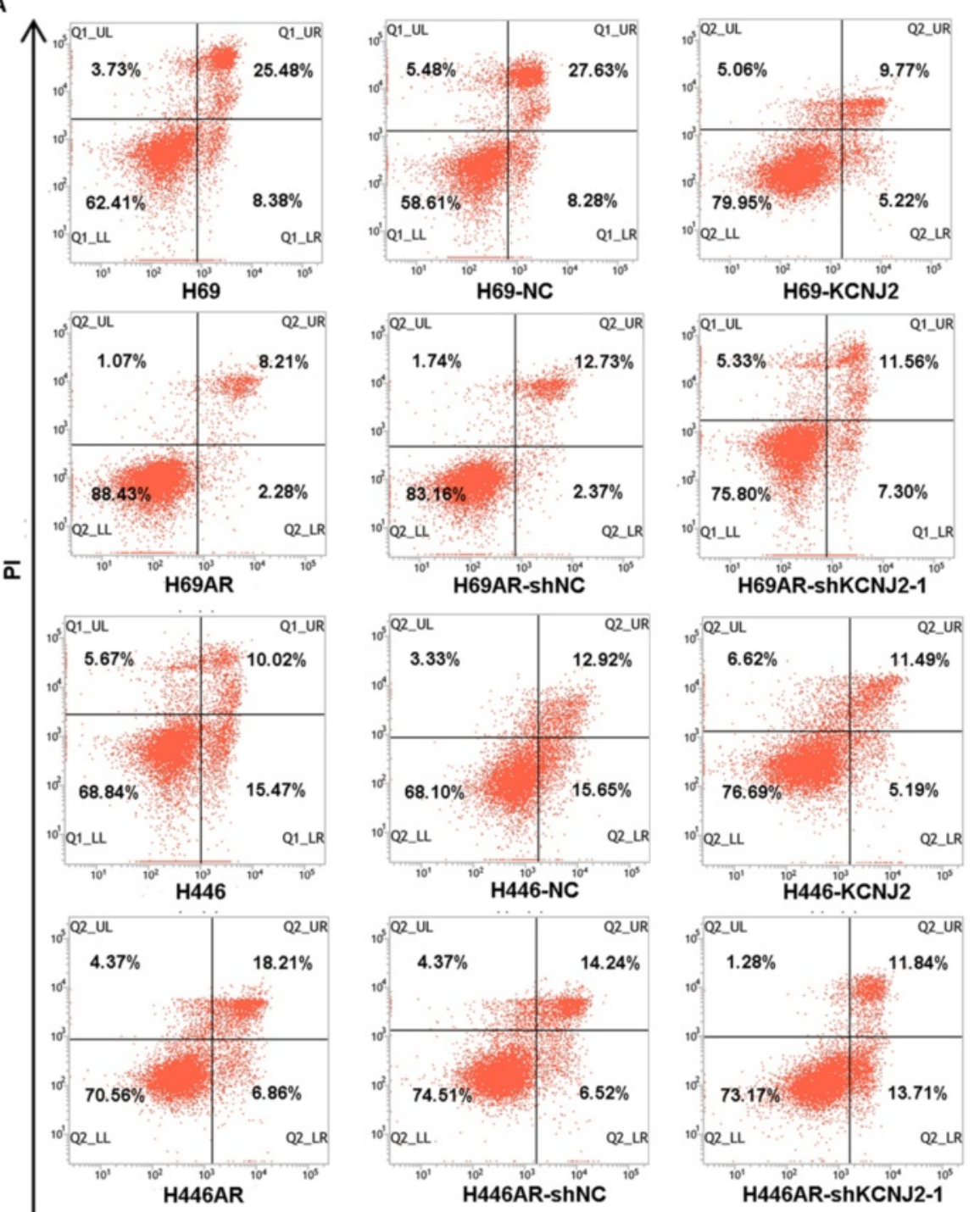

Annexin V

B

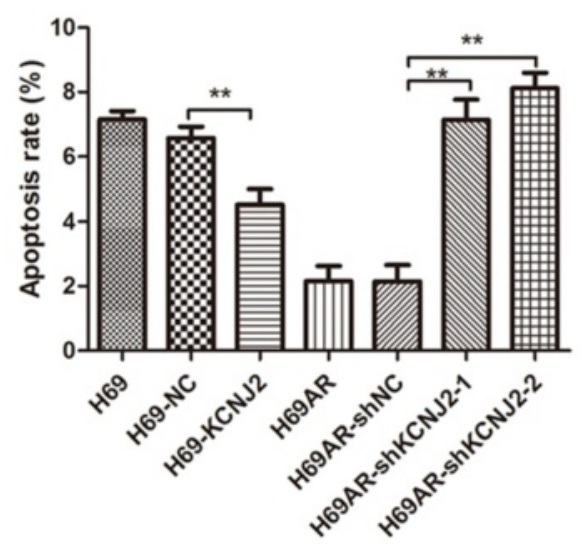

Figure 5 (See legend on next page.)
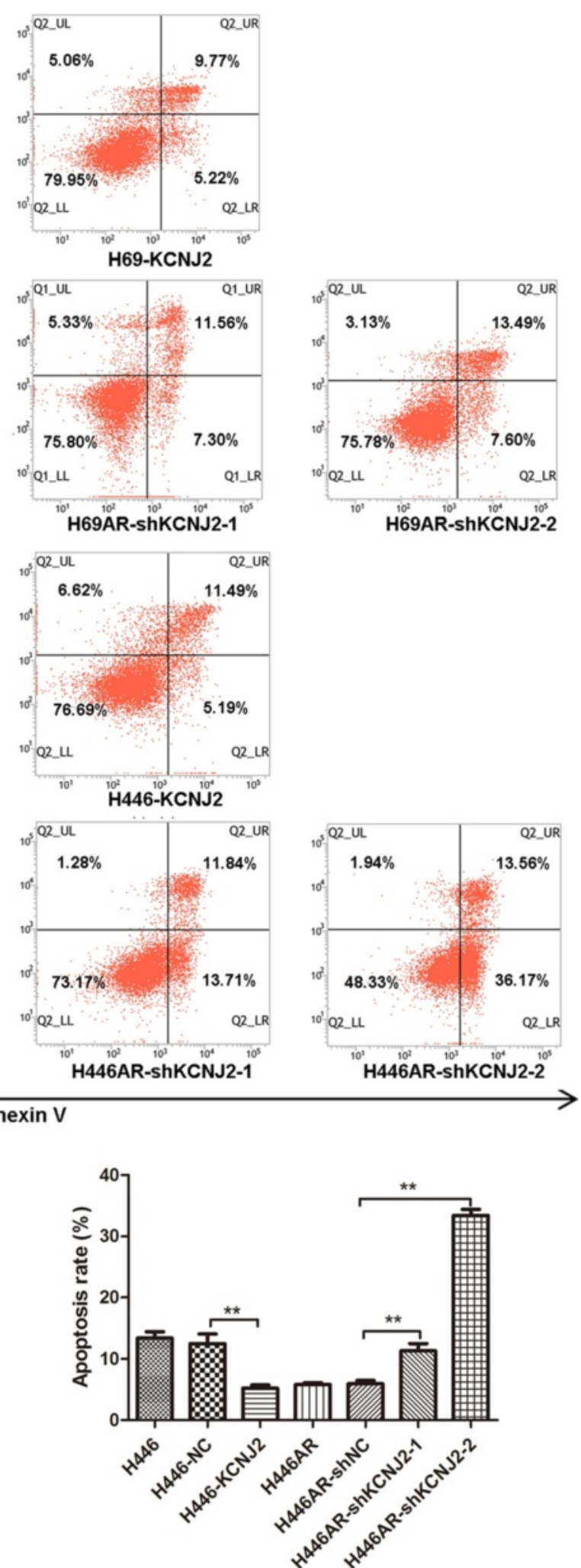
(See figure on previous page.)

Figure 5 KCNJ2/Kir2.1 affects cell apoptosis in SCLC cells after ADM treatment. (A) Cells were analyzed by annexin-V/propidium iodide (PI) dual labeling. Representative FACS profiles are shown, on which cell population in the quadrant of Annexin $\mathrm{V}^{+} \mathrm{PI}^{-}$represents apoptotic cells. (B) Histographs for cell apoptosis of SCLC cells. The percentage of the Annexin $\mathrm{V}^{+} \mathrm{PI}^{-}$cell population was determined. The results show data from at least three independent experiments. ${ }^{*}, P<0.01$ compared with the corresponding NC cells.

\section{Discussion}

Previous studies have revealed that $\mathrm{K}+$ channel blockers inhibit SCLC cell proliferation via membrane depolarization $[16,28]$ and that some types of inwardly rectifying $\mathrm{K}^{+}$channels are involved in SCLC MDR $[16,29]$. In our previous analysis of a cDNA microarray [22], KCNJ2 expression was increased in SCLC multidrug resistant H69AR cells compared with the parent H69 cell line, suggesting that KCNJ2/Kir2.1 might be relevant in the drug resistance of SCLC. However, the molecular mechanism by which KCNJ2/Kir2.1 exerts a role in the chemoresistance of SCLC was not clear until now. In this study, we showed that the mRNA and protein expression of $\mathrm{KCNJ} 2 / \mathrm{Kir} 2.1$ was upregulated in both H69AR and H446AR cells compared with that in their respective parental cells, confirming the results of the SCLC mRNA expression profiling. Moreover, Kir2.1 expression in 52 clinical SCLC tissues was significantly associated with the chemotherapeutic responses of the SCLC patients. To further investigate whether KCNJ2/ Kir2.1 regulates MDR, we first established two stable $\mathrm{KCNJ} 2 /$ Kir2.1-overexpressing sublines, H69-KCNJ2 and

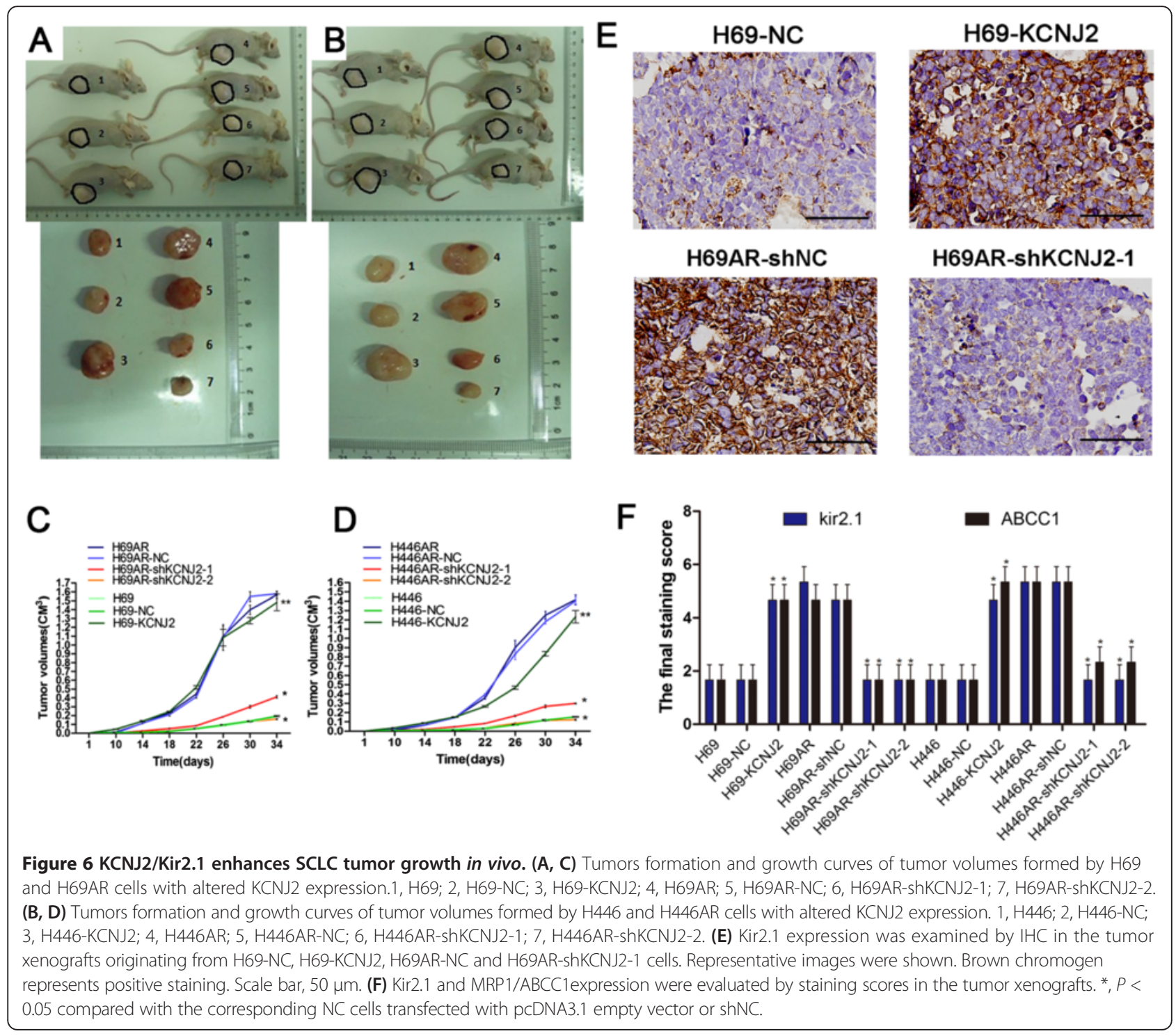


Table 3 The correlation between Kir2.1 and MRP/ABCC1 in SCLC tissues

\begin{tabular}{|c|c|c|c|c|}
\hline \multirow[t]{2}{*}{$\mathrm{ABCC} 1$} & \multicolumn{2}{|c|}{ Kir2.1 } & \multirow[t]{2}{*}{$x^{2}$} & \multirow[t]{2}{*}{$P$} \\
\hline & - & + & & \\
\hline- & 23 & 2 & 25.622 & $<0.001$ \\
\hline+ & 6 & 21 & & \\
\hline
\end{tabular}

The P-value was calculated by $x^{2}$ test.
H446-KCNJ2, and suppressed KCNJ2/Kir2.1 expression in H69AR and H446AR cells by shRNAs specifically targeting KCNJ2. Next, we examined the effect of KCNJ2/Kir2.1 upregulation and downregulation on the sensitivity of SCLC cells to chemotherapeutic drugs (ADM, CDDP and VP-16). H69AR and H446AR cells became much more sensitive to chemotherapeutic agents than the NC groups after significantly inhibiting KCNJ2/Kir2.1 expression, whereas $\mathrm{KCNJ} / \mathrm{Kir} 2.1$ upregulation led to the desensitization of $\mathrm{H} 69$ and $\mathrm{H} 446$ cells to these drugs.
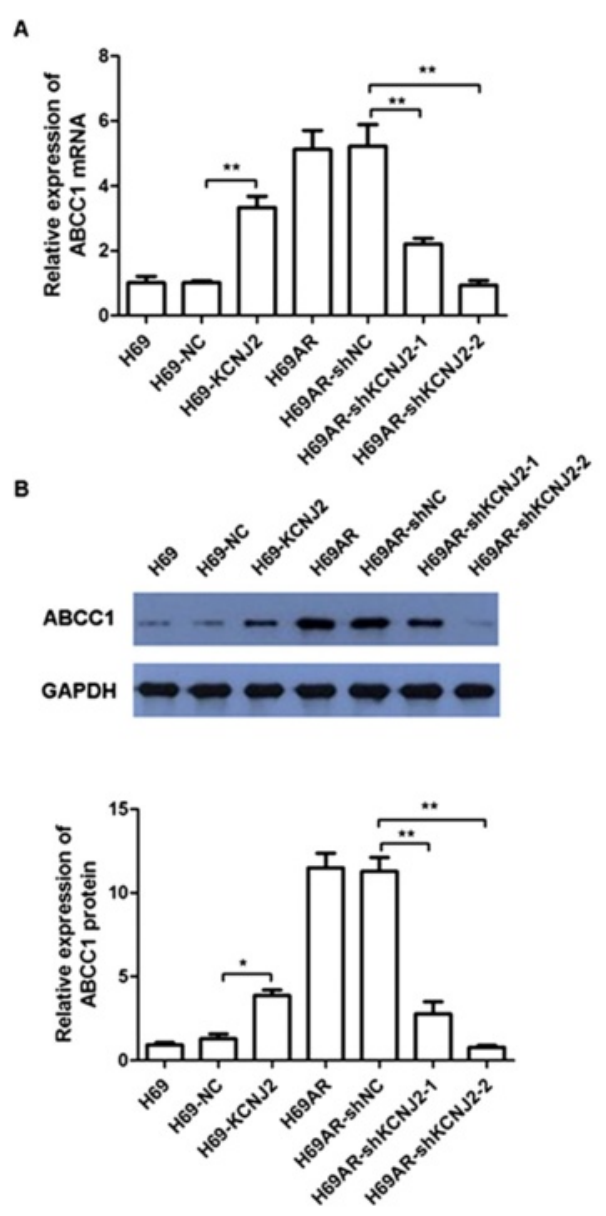

C

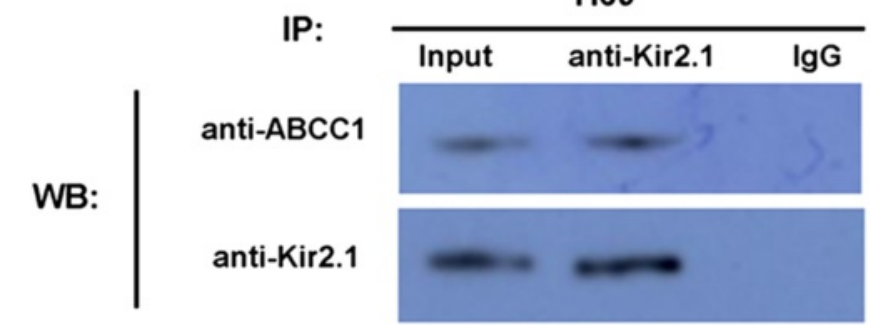

Figure 7 Kir2.1 interacts with MRP1/ABCC1 expression. (A-B) qRT-PCR (A) and Western blotting (B) analyses showed that KCNJ2/Kir2.1 upregulation or downregulation successfully elevated or suppressed MRP1/ABCC1 mRNA and protein levels, respectively. (C) The interaction between Kir2.1 and MRP1/ABCC1 was analyzed by Co-IP. Anti-Kir2.1 antibody was used for IP. The amounts of ABCC1 and Kir2.1 in the immunoprecipitates were detected by Western blotting with the indicated specific antibodies. 
A

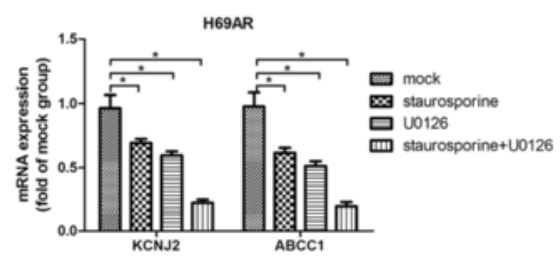

B
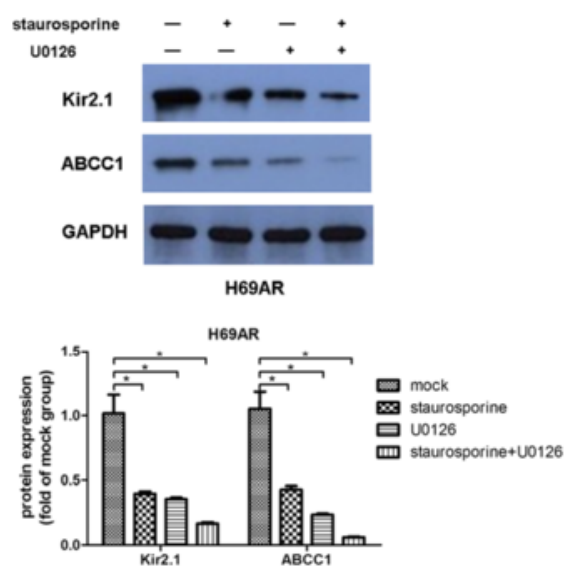
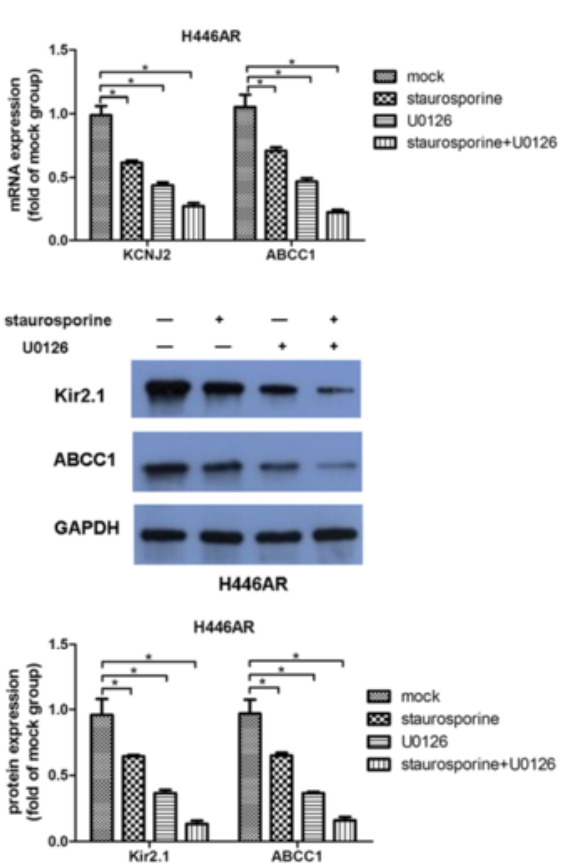

Figure 8 The Ras/MAPK pathway modulates the expression of KCNJ2/Kir2.1. (A-B) qRT-PCR and Western blot analyses showed that KCNJ2/ Kir2.1 expression was inhibited at the mRNA and protein levels in H69AR and H446AR cells treated with the PKC inhibitor staurosporine, the MEK inhibitor U0126, or staurosporine plus U0126. *, $P<0.05$ compared with the corresponding mock cells.

Our findings indicate that $\mathrm{KCNJ} 2 / \mathrm{Kir} 2.1$ is closely correlated with chemoresistance and may represent a potential clinical strategy for interfering with chemoresistance in SCLC; however, more clinical data are needed to verify this proposal.

To further investigate the possible mechanism of KCNJ2/Kir2.1 in SCLC chemoresistance, we evaluated the effect of KCNJ2/Kir2.1 on apoptosis and cell cycle control by flow cytometry. Our results indicated that one reason for the resistant phenotype of MDR SCLC cells may be that KCNJ2/Kir2.1 induces cell cycle arrest at the G0/G1 phase and inhibits drug-induced apoptosis. Moreover, consistent with the results obtained in vitro, KCNJ2/Kir2.1 promoted tumor growth in a xenograft nude mouse model. These results suggest that KCNJ2/ Kir2.1 may play an oncogenic role in SCLC.

In addition to the potential therapeutic impact of KCNJ2/Kir2.1, our studies shed light on the mechanisms by which KCNJ2/Kir2.1 mediates MDR in SCLC. Several studies have confirmed that MRP1/ABCC1 is highly expressed in H69AR cells [30,31], and may be closely related to chemoresistance in SCLC [22]. Enyeart et al. showed that $\mathrm{K}^{+}$channels, including $\mathrm{KCNJ} 2 / \mathrm{Kir} 2.1$, might function with MRP1/ABCC1 [32]. In this study, we first found that MRP1/ABCC1 expression was positively correlated with KCNJ2/Kir2.1in SCLC cells and tissues. To further confirm this relationship, we performed Co-IP and demonstrated that Kir2.1 can interact with MRP1/
ABCC1 in H69AR cells. Our data suggest that KCNJ2/ Kir2.1 might affect the resistance to chemotherapy via interaction with MRP1/ABCC1 in SCLC cells.

The KCNJ2/Kir2.1 channel is modulated by several factors, including PKC, direct tyrosine kinase phosphorylation, the acidic intracellular $\mathrm{pH}$ and AMP-activated protein kinase [17,33-35]. The work reported by Giovannardi et al. has shown that RAS-PKC-MEK signaling is also an important regulator of $\mathrm{KCNJ} 2 / \mathrm{Kir} 2.1$ [5]. In this study, $\mathrm{KCN}$ 2/Kir2.1 expression at the mRNA and protein levels was markedly downregulated in the H69AR and H446AR cells after treatment with staurosporine, a PKC inhibitor, or U0126, a MEK inhibitor. It has been suggested that the KCNJ2/Kir2.1 channel is regulated by the Ras-MAPK pathway. However, whether Ras-MAPK signaling is involved in the mechanism by which KCNJ2/Kir2.1regulates SCLC MDR remains unknown.

miRNAs play an important role in the development of drug resistance in some tumor types [24,36], and our previous study showed that some miRNAs are involved in the development of drug resistance in SCLC cells [22]. Thus, we hypothesized that certain miRNAs could affect chemosensitivity by directly targeting KCNJ2/ Kir2.1 in SCLC, and we identified miR-7 as a direct suppressor of KCNJ2/Kir2.1. Recently, miR-7 was reported to be a tumor suppressor due to its abilities to suppress cell growth and metastasis [37-39], promote apoptosis and inhibit drug resistance [40]. Our data showed that 


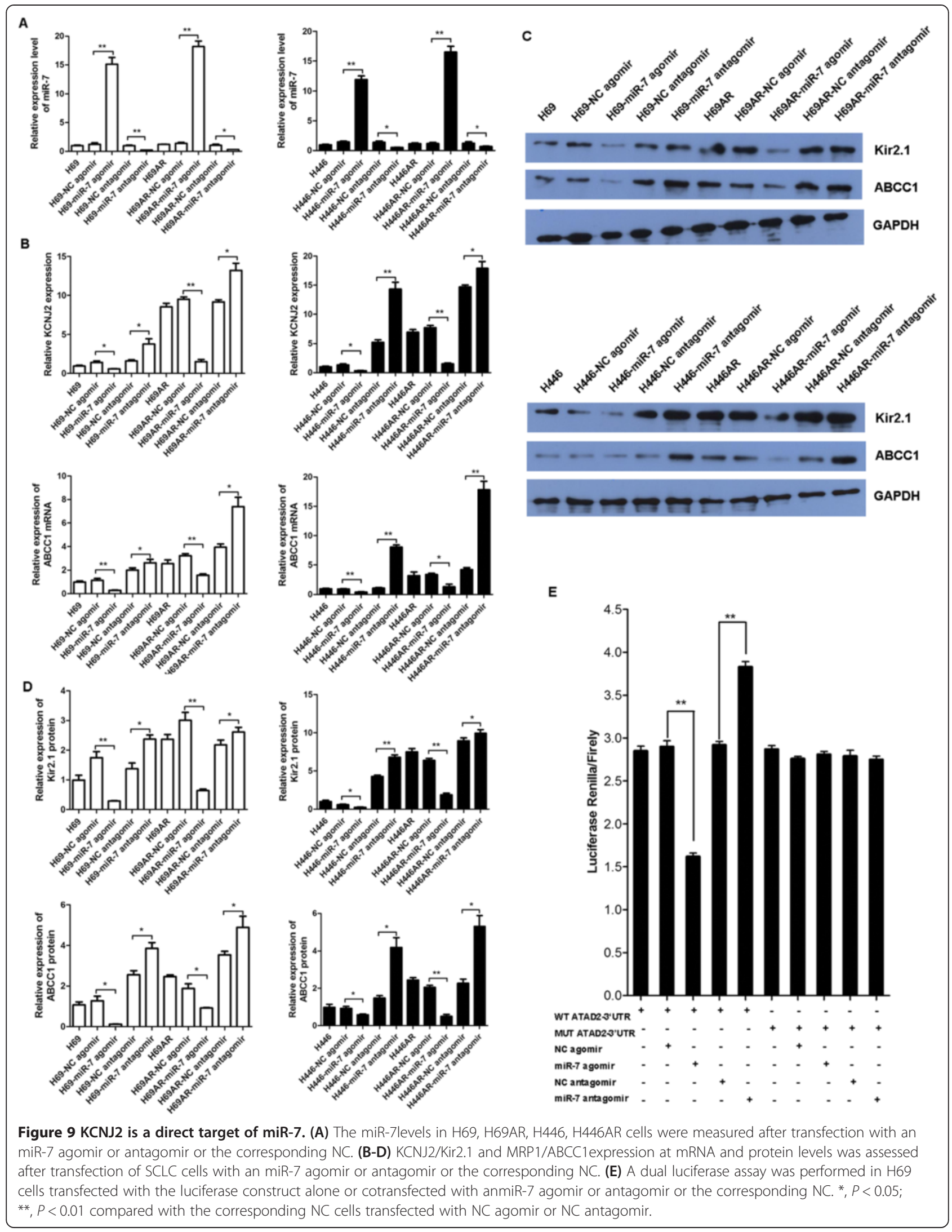


A

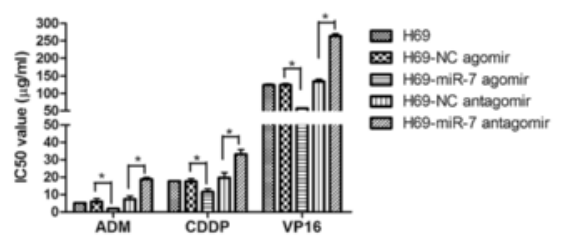

C

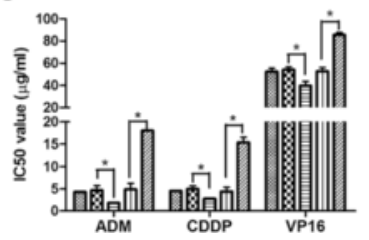

B

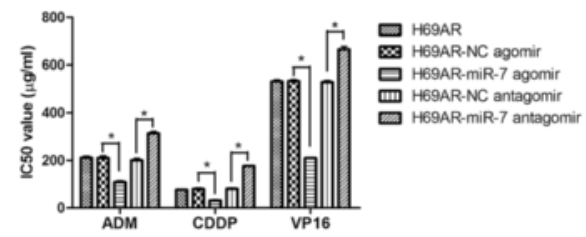

D

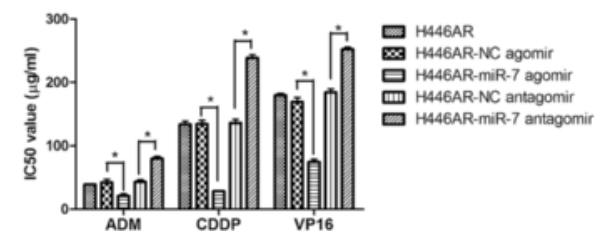

Figure 10 The effect of miR-7 on drug sensitivity. (A-B) The sensitivity of H69 and H69AR cells to chemotherapeutic drugs (ADM, CDDP and VP-16) after transfected with the miR-7 agomir, antagomir or the corresponding NC. (C-D) The sensitivity of H446 and H446AR cells to chemotherapeutic drugs (ADM, CDDP and VP-16) after transfected with the miR-7 agomir, antagomir or the corresponding NC. ADM, Adriamycin; CDDP, Cisplatin; VP-16, Etoposide. ${ }^{*} P<0.05$ (compared with corresponding negative control groups).

an miR-7 agomir or an antagomir led to a significant decrease or increase, respectively, in $\mathrm{KCNJ} 2 / \mathrm{Kir} 2.1$ expression at both the mRNA and protein levels in SCLC cells. Luciferase reporter assays demonstrated that miR-7 directly targeted KCNJ2 in H69AR cells, and miR-7 expression was associated with SCLC chemoresistance. In addition, we found that miR-7 downregulation was associated with $\mathrm{KCNJ} 2 / \mathrm{Kir} 2.1$ expression and advanced clinicopathological features of SCLC tissues. These findings indicate that KCNJ2/Kir2.1 is directly regulated by miR-7 in SCLC.

In summary, our findings reported here provide a novel mechanism by which KCNJ2/Kir2.1 modulates the sensitivity of SCLC cells to chemotherapeutic drugs,

Table 4 Relationship between miR-7 expression and clinicopathological characteristics in SCLC patients

\begin{tabular}{|c|c|c|c|c|c|}
\hline \multirow[t]{2}{*}{ Characteristics } & \multirow[t]{2}{*}{ Total } & \multicolumn{2}{|c|}{ MiR-7 expression } & \multirow[t]{2}{*}{$x^{2}$} & \multirow[t]{2}{*}{$P$ value } \\
\hline & & - & + & & \\
\hline Gender & & & & 0.134 & 0.714 \\
\hline Male & 43 & 22 & 21 & & \\
\hline Female & 9 & 4 & 5 & & \\
\hline Age & & & & 0.115 & 0.734 \\
\hline$<55^{\dagger}$ & 11 & 6 & 5 & & \\
\hline$\geq 55$ & 41 & 20 & 21 & & \\
\hline Stage & & & & 6.240 & 0.012 \\
\hline Limited disease & 25 & 8 & 17 & & \\
\hline Extensive disease & 27 & 18 & 9 & & \\
\hline Drug-sensitivity & & & & 3.733 & 0.053 \\
\hline Sensitive group & 25 & 10 & 15 & & \\
\hline Resistant group & 22 & 15 & 7 & & \\
\hline Kir2.1 & & & & 13.175 & $<0.001$ \\
\hline Negative & 29 & 8 & 21 & & \\
\hline Positive & 23 & 18 & 5 & & \\
\hline $\mathrm{ABCC} 1$ & & & & 9.321 & 0.002 \\
\hline Negative & 25 & 7 & 18 & & \\
\hline Positive & 27 & 19 & 8 & & \\
\hline
\end{tabular}

${ }^{\dagger}$ median age.

-, low expression; + , high expression.

The P-value was calculated by $x^{2}$ test 
possibly through its regulation of MRP1/ABCC1 and simultaneous regulation by the Ras/MAPK pathway and miR-7. Therefore, our study indicates that KCNJ2/Kir2.1 may be a potential novel target for interfering with chemoresistance in SCLC.

\section{Methods and materials Tissue specimens}

Fifty-two SCLC patient tissue samples were obtained from Zhujiang (Southern Medical University, Guangzhou, China) and Wujing Hospitals (Guangzhou Medical University, Guangzhou, China). All samples were confirmed as SCLC by pathologic examination and were further distinguished as limited disease (25 cases) or extensive disease (27 cases) according to the Veterans Administration Lung Study Group. All patients gave informed consent prior to the collection of specimens according to the institutional guidelines. Tissue samples were snap-frozen in the operating room immediately after surgery, and non-tumor tissues were sent to the pathology department for diagnosis by a board-certified pathologist. The non-tumor tissues (pericarcinomatous tissues) were confirmed to have surrounded the tumor tissue and to be free of cancer cells. A paraffin-embedded tissue specimen was available for each included patient. Under the protocol approved by the Institutional Review Board, informed consent was obtained from the patients or their guardians.

\section{Cells culture and transfection}

Human SCLC H69 and H446 cell lines and the drugresistant H69ARsubline were purchased from American Type Culture Collection (ATCC, USA). The other drugresistant subline, H446AR, was established in our laboratory by culturing $\mathrm{H} 446$ cells in adriamycin (ADM). These cell lines were maintained in RPMI 1640 (GIBCO, Mississauga, Canada) supplemented with 10\% heatinactivated calf serum (HyClone, Logan, UT) and Lglutamine (Beyotime, Jiangsu, China) in an incubator at $37^{\circ} \mathrm{C}$ with $5 \% \mathrm{CO}_{2}$. The H69AR and $\mathrm{H} 446 \mathrm{AR}$ cell lines were challenged monthly for maintained resistance to the selected drugs, and their growth and morphology were monitored. The drug-resistant cells were maintained in drug-free medium for at least 2 weeks before any experiment.

For transient miRNAtransfection, cells were placed in standard media without antibiotics for $24 \mathrm{~h}$ before being transfected with anmiR-7agomir or antagomir or the corresponding negative controls (GenePharma, Shanghai, China) using Lipofectamine 2000 and OPTI-MEMI (Invitrogen) according to the manufacturer's protocol. For stable transfections, the KCNJ2 coding region was inserted into pcDNA3.1 (GenePharma) andtransfected into H69 and H446 cells to stably overexpress KCNJ2 (H69-KCNJ2 and H446-KCNJ2). Cells stably transfected with the pcDNA3.1 empty expression vector (Invitrogen) were used as their corresponding negative controls (H69-NC and $\mathrm{H} 446-\mathrm{NC}$ ). Positive transfectants were selected with $800 \mu \mathrm{g} / \mathrm{ml}$ geneticin (G418; Invitrogen). The KCNJ2 gene was knocked down using two different KCNJ2 short-hairpin RNAs (shKCNJ2-1, shKCNJ2-2), which were obtained from GenePharma and transfected into H69AR and H446AR cells (H69AR-shKCNJ2-1, H69AR-shKCNJ2-2, H446AR-shKCNJ2-1 and H446ARshKCNJ2-2) using Lipofectamine 2000. A negative control shRNA (shNC) was transfected into H69AR and H446AR cells (H69AR-shNC and H446AR-shNC) as the corresponding negative controls for cells transfected with shKCNJ2-1 or shKCNJ2-2. After the cells were treated for 24 h, G418 was used for 1 month to select the transfected cells. The short-hairpin RNAsequences are shown in Additional file 7: Table S2.

\section{Reagents and antibodies}

A rabbit anti-human Kir2.1 polyclonal antibody was purchased from Alomone labs (Jerusalem, Israel), andpolyclonal anti-MRP1/ABCC1 and glyceraldehyde3-phosphate dehydrogenase (GAPDH) monoclonal antibodies were purchased from Santa Cruz Inc. (CA, USA). Horseradish peroxidase (HRP)-labeled goat antirabbit immunoglobulin G (IgG) and goat anti-mouse IgG were obtained from Santa Cruz Inc. U0126, the MEK inhibitor, and staurosporine, the PKC inhibitor, were purchased from Selleck Chemicals (Houston, TX, USA). All three chemotherapeutic drugs, Cisplatin (DDP; Shandong, China), Etoposide (VP-16; Jiangsu, China) and Adriamycin (ADM; Jiangsu, China), were obtained from commercial sources and were dissolved according to the manufacturer's instructions.

\section{RNA isolation, reverse transcription, and quantitative real-time PCR}

Total RNA, including miRNA, was extracted from cell lines using TRIzol (Invitrogen) or the miRNeasy kit (Qiagen), according to the manufacturer's instructions. For formalin-fixed, paraffin-embedded (FFPE) samples, total RNA was extracted from ten to fifteen $10-\mu$ m-thick sections usingmiRNeasy FFPE Kit (Qiagen). Total RNA was reverse transcribed using the PrimeScript RT reagent Kit (Takala, Dalian, China), andmiRNA sequence-specific reverse transcription (RT)-PCR for miR-7 and U6 was performed according to the Hairpin-itTMmiRNAs q-PCR quantitation kit and the U6 snRNA real-time PCR normalization kit (GenePharma). Quantitative real-time PCR was carried out using the MX3005 sequence detection system (Stratagene) with SYBR Green according to the manufacturer's instructions. All primers are listed in Additional file 7: Table S3. GAPDH and U6 were used as 
endogenous controls. All samples were normalized to the internal controls, and fold changes were calculated through relative quantification $\left(2^{-\triangle \Delta C t}\right)[41]$.

\section{Western blotting assay}

For western blotting assays, total proteins were extracted from cells using RIPA lysis buffer (Sigma-Aldrich) and quantified using a BCA protein assay kit (Thermo). Total proteins were separated on $8 \%$ SDS-PAGE gels before being transferred to polyvinylidenedifluoride membranes (Bio-Rad). After the membranes were blocked with $5 \%$ non-fat milk, they were incubated with a rabbit anti-human Kir2.1 polyclonal or mouse antihuman MRP1/ABCC1 polyclonal antibody at $4^{\circ} \mathrm{C}$ overnight. GAPDH was used as a protein-loading control. After washing with Tris-buffered saline solution containing $0.1 \%$ Tween 20 (TBST, Bio-Rad), a peroxidase-linked secondary goat anti-mouse IgG or goat anti-rabbit IgG antibody was incubated with the membranes for $1 \mathrm{~h}$ at room temperature. After washing again with TBST, the protein bands were detected by chemiluminescence. The intensities of the protein bands were quantified with the Quantity One software (4.5.0 basic, Bio-Rad).

\section{Immunofluorescence staining}

Cells were seeded into 24-well plates for $24 \mathrm{~h}$ before being fixed with paraformaldehyde at $4{ }^{\circ} \mathrm{C}$ for $30 \mathrm{~min}$. After being rinsed in PBS, the cells were incubated with $10 \%$ normal calf serum for 30 min to block non-specific IgG binding sites and then incubated with rabbit anti-human Kir2.1 monoclonal antibody (Alomone) (1:100 dilution) at $4^{\circ} \mathrm{C}$ overnight. A fluorescein isothiocyanate (FITC)-conjugated goat anti-rabbit IgGsecondary antibody (1:100 dilution) was added, and the cells were incubated for $1.5 \mathrm{~h}$ in the dark at room temperature. All images were captured using a fluorescence microscope (model Eclipse 660, Nikon, Japan).

\section{In vitro drug sensitivity assay}

Cells were reseeded in 96-well plates at a density of $5 \times$ $10^{3}$ per well and treated in medium with ADM, DDP or VP-16 for $24 \mathrm{~h}$. Cell survival was then analyzed via the Cell Counting Kit-8 assay (CCK8, Dojindo Molecular Technologies, Japan) according to the manufacturer's instructions. The range of drug concentrations was based on earlier studies [42] and was aimed to obtain $\mathrm{IC}_{50}$ values for both highly sensitive and resistant cases. After incubation with $10 \mu \mathrm{l}$ of CCK- 8 reagent for $4 \mathrm{~h}$,the absorbance was measuredat $450 \mathrm{~nm}$. The cells incubated without drugs were set at $100 \%$ survival and used to calculate the $\mathrm{IC}_{50}$ of each chemotherapeutic drug. The assay was carried out in six replicate wells for each sample, and three parallel experiments were conducted.

\section{Flow cytometric analysis}

Cells were treated with drugs for $24 \mathrm{~h}$ and then collected for apoptosis and cell cycle analyses. Cell apoptosis assays were performed using an Annexin V/propidium iodide (PI) detection kit (Keygene, Nanjing, China) according to the manufacturer's instructions. For cell cycle analysis, cells were harvested and fixed in $70 \%$ ethanol overnight at $4^{\circ} \mathrm{C}$. After being washed three times in cold PBS, the cells were incubated with RNase and stained with PI. Cellquest Pro software was used for apoptosis analysis and ModFit LT software was used for analysis of cell cycle. Cells in the quadrant of Annexin $\mathrm{V}^{-} \mathrm{PI}^{-}$(lower left) represent viable cells, cells in the quadrant of Annexin $\mathrm{V}^{-} \mathrm{PI}^{+}$(upper left) mean necrotic cells, cells in the quadrant of Annexin $\mathrm{V}^{+} \mathrm{PI}^{+}$(upper right) mean late apoptotic and dead cells and cells in the quadrant of Annexin $\mathrm{V}^{+} \mathrm{PI}^{-}$(lower right) represent early apoptotic cells. Usually apoptosis analysis is mainly based on the percentages of Annexin $\mathrm{V}^{+} \mathrm{PI}^{-}$cells. All assays were carried out independently in triplicate.

\section{Immunohistochemical analysis}

Paraffin-embedded SCLC samples were sectioned and mounted on microscopic slides. Polyclonal anti-Kir2.1 and anti-MRP1/ABCC1 antibodies (Alomone Labs Ltd., Israel) was used as the primary antibodies. Antigen retrieval was performed by microwaving in $10 \mathrm{mmol} / \mathrm{L}$ citric acid buffer at $\mathrm{pH}$ 7.2. The samples were incubated first with the primary antibodies overnight at $4^{\circ} \mathrm{C}$, then with the secondary antibodies for $2 \mathrm{~h}$ at room temperature in the same buffer and finally with abiotinylated secondary antibody (DAKO, Tokyo, Japan). The bound antibodies were visualized using the avidinbiotinylated peroxidase complex and diaminobenzidine tetrachloride methods (Santa Cruz Biotechnology).

The IHC-stained samples were independently evaluated for Kir2.1 and ABCC1 expressions by two pathologists blinded to the clinical parameters. The staining intensity was scored as 0 (negative), 1 (weak), 2 (medium) or 3 (strong). The extent of staining was scored as $0(0-5 \%), 1(6-25 \%), 2(26-50 \%)$ or 3 (51$100 \%)$, according to the percentages of the positive stained area in relation to the entire carcinomainvolved area or the entire normal sample area. The sum of the intensity and extent scores was used as the final staining score $(0-6)$. Optimal cut off values were identified; a final staining score $\leq 1$ indicated negative expression and a final staining score $>1$ indicated positive expression.

\section{Luciferase reporter assay}

The wildtype and mutated KCNJ23'UTR segments that were predicted to interact with miR-7 were amplified from human genomic DNA by PCR and inserted into 
psiCHECK-2 immediately downstream of the luciferase stop codon of (Promega) to develop psiCHECK2-KCNJ2-3' UTR and psiCHECK2-KCNJ2-mut-3'UTR. Cells in 24-well plates were transfected with psiCHECK2-KCNJ2-3'UTR, psiCHECK2-KCNJ2-mut-3'UTR or psiCHECK-2. Moreover, anmiR-7 agomir or antagomiror their corresponding negative control (NC agomir or $\mathrm{NC}$ antagomir) was also co-transfected into the cells. Luciferase activity was then assayed $48 \mathrm{~h}$ posttransfection using a dual-luciferase reporter assay system (Promega).

\section{Co-immunoprecipitation}

Cells cultured in $10-\mathrm{cm}$ dishes were harvested and lysed in $500 \mu$ l lysis buffer (20 mMTris (pH 7.4), 50 $\mathrm{mMNaCl}, 1 \mathrm{mM}$ EDTA, 0.5\% NP-40, 0.5\% SDS, 0.5\% deoxycholate, and protease inhibitors). Then, $500 \mu \mathrm{g}$ lysate $(1 \mu \mathrm{g} / \mu \mathrm{l})$ was precleared with $50 \mu \mathrm{l}$ protein ASepharose beads (Upstate Biotechnology, NY, USA) for $2 \mathrm{~h}$ at $4^{\circ} \mathrm{C}$. An appropriate amount of rabbit anti-Kir2.1 antibody (Alomone) or rabbit non-specific IgG (Santa) was then added and incubated overnight at $4^{\circ} \mathrm{C}$. Then, $100 \mu \mathrm{l}$ preblocked agarose beads was added to the antibody/lysate mixture and incubated for another $2 \mathrm{~h}$ at $4^{\circ} \mathrm{C}$; the beads were then pelleted and washed twice with lysis buffer. Both the cell lysates without IP and the immunoprecipitates were eluted in SDS sample buffer, subjected to SDS-PAGE and analyzed by immunoblotting.

\section{In vivo tumor xenograft model}

Six- to eight-week-old female BALB/c nude mice (purchased from the Medical Experimental Animal Center of Guangdong Province, China) were used for in vivo assays. The mice were raised under pathogen-free conditions, and all procedures were performed according to the guidelines of the Association for the Assessment and Accreditation of Laboratory Animal Care International. Cells were harvested, washed with PBS and re-suspended in normal culture medium at a concentration of $1 \times 10^{7}$ cells $/ 0.1 \mathrm{ml}$. Cells in RPMI 1640 were subcutaneously inoculated into the legs of nude mice to establish the tumor model. The tumor volume was determined three times per week by direct measurement with a sliding caliper and was calculated using the following equation: $V=(4 / 3) \times \pi \times R 1^{2} \times R 2$, where $\mathrm{R} 1$ is radius $1, \mathrm{R} 2$ is radius 2 , and $\mathrm{R} 1<\mathrm{R} 2$. Growth curves of the tumors were constructed. After 20 days, 5 mice from each group were sacrificed, and the tumors were excised and fixed with neutral phosphate-buffered formalin. Subsequently, consecutive tissue sections from the tumors were sliced and then stained with hematoxylin-eosin.

\section{Statistical analysis}

All experiments were run in triplicate, and the results are presented as the mean \pm SD. Statistical analyses were performed using either an analysis of variance (ANOVA) or Student's $t$ test. The association between Kir2.1or MRP1/ABCC1 or miR-7expression and clinical features was analyzed by $\chi^{2}$ test. The relationship between Kir2.1 and MRP1/ABCC1 was explored by $\chi^{2}$ test. Survival curves were obtained by Kaplain-Meier analysis. The positive rate of Kir2.1 and MRP1/ABCC1 in normal lung tissue was compared with that in SCLC tissue by $\chi^{2}$ test. A difference was considered statistically significant when the $\mathrm{P}$ value was less than 0.05 . All statistical analyses were carried out with SPSS 17.0 software.

\section{Additional files}

\section{Additional file 1: Figure S1. The semiquantitative analyses of $\mathrm{IHC}$ for Kir2.1 and ABCC1 expression. (A) The final staining scores of $\mathrm{IHC}$ in Figure 1A-D for Kir2.1 and ABCC1 expression in SCLC and normal lung tissues. (B) The final staining scores of $\mathrm{HC}$ in Figure 6E for Kir2.1 expression.}

Additional file 2: Figure S2. Altered expression of KCNJ2/Kir2.1 affects the cell cycle distribution of SCLC cells after treatment with CDDP.

(A) Representative FACS profiles are shown, on which numbers indicate percentage of cells in G0/G1, S or G2/M phase. (B) The histographs for cell cycle distribution of SCLC cells. Data are shown as means \pm SD from three independent experiments. ${ }^{*}, P<0.05 ;{ }^{*}, P<0.01$ compared to the corresponding NC cells transfected with pcDNA3.1 empty vector or shNC.

Additional file 3: Figure S3. Altered expression of KCNJ2/Kir2.1 affects the cell cycle distribution of SCLC cells after treatment with VP-16.

(A) Representative FACS profiles are shown, on which numbers indicate percentage of cells in G0/G1, S or G2/M phase. (B) The histographs for cell cycle distribution of SCLC cells. Data are shown as means \pm SD from three independent experiments. ${ }^{*}, P<0.05$; ${ }^{* *}, P<0.01$ compared to the corresponding NC cells transfected with pcDNA3.1 empty vector or shNC.

Additional file 4: Figure S4. Altered expression of KCNJ2/Kir2.1 affects cell apoptosis in SCLC cells after treatment with CDDP. (A) Representative FACS profiles are shown. (B) The histographs for cell apoptosis of SCLC cells. The results show data from at least three independent experiments. *, $P<0.05$; ${ }^{* *}, P<0.01$ compared to the corresponding NC cells.

Additional file 5: Figure S5. Altered expression of KCNJ2/Kir2.1 affects cell apoptosis in SCLC cells after treatment with VP-16. (A) Representative FACS profiles are shown. (B) The histographs for cell apoptosis of SCLC cells. The results show data from at least three independent experiments. ${ }^{*}, P<0.05 ;{ }^{* *}, P<0.01$ compared to the corresponding NC cells.

Additional file 6: Figure S6. Kaplan-Meier analysis of overall survival of 52 patients with SCLC based on miR-7 expression. $(P<0.05)$

Additional file 7: Table S1. Univariate analysis of overall survival with regard to clinicopathological characteristics. Table S2. The sequences of short hairpin RNA used in vector construction. Table S3. Primers used in real-time quantitative RT-PCR.

\section{Abbreviations}

IHC: Immunohistochemistry; MRP1/ABCC1: Multidrug resistance protein 1; MDR: Multidrug resistance; MiRNAs: microRNAs; GAPDH: Glyceraldehyde-3phosphate dehydrogenase.

\section{Competing interests}

The authors declare that they have no competing interests. 


\section{Authors' contributions}

$\mathrm{HL}$ carried out the experimental work and drafted the manuscript. JH performed the statistical analysis and drafted the manuscript. JP collected clinical specimens and participated in the molecular biology studies. XW participated in cell proliferation assays and animal experiments. $Y Z$ helped to carry out the immunohistochemistry assays. WZ participated in the collection of clinical specimens. LG designed the study and critically revised the manuscript. All authors read and approved the final manuscript.

\section{Acknowledgements}

We thank Professor Song-Shan Tang from Guangdong Pharmaceutical University for his careful revision of the English grammar in this manuscript. This work was supported by the National Natural Science Foundation of China (81172241) and the Guangdong Provincial Natural Science Fund (S2011010004065).

\section{Author details}

'Department of Pathology, Zhujiang Hospital, Southern Medical University, Guangzhou, China. ${ }^{2}$ Department of Pathology, Guangdong Provincial Corps Hospital of Chinese People's Armed Police Forces, Guangzhou Medical College, Guangzhou, China. ${ }^{3}$ Department of Pathology, the Third Affiliated Hospital of Guangzhou Medical University, Guangzhou, China. ${ }^{4}$ Department of Oncology, Zhujiang Hospital, Southern Medical University, Guangzhou, China.

\section{Received: 6 September 2014 Accepted: 15 January 2015}

Published online: 12 March 2015

\section{References}

1. Parkin DM, Bray F, Ferlay J, Pisani P. Global cancer statistics, 2002. CA Cancer J Clin. 2005;55(2):74-108.

2. Planchard D, Le Pechoux C. Small cell lung cancer: new clinical recommendations and current status of biomarker assessment. Eur J Cancer. 2011:47 Suppl 3:S272-83

3. Roberti A, La Sala D, Cinti C. Multiple genetic and epigenetic interacting mechanisms contribute to clonally selection of drug-resistant tumors: current views and new therapeutic prospective. J Cell Physiol. 2006;207(3):571-81.

4. Yeh JJ, Hsu NY, Hsu WH, Tsai CH, Lin CC, Liang JA. Comparison of chemotherapy response with P-glycoprotein, multidrug resistance-related protein-1, and lung resistance-related protein expression in untreated small cell lung cancer. Lung. 2005;183(3):177-83.

5. Giovannardi S, Forlani G, Balestrini M, Bossi E, Tonini R, Sturani E, et al. Modulation of the inward rectifier potassium channel IRK1 by the Ras signaling pathway. J Biol Chem. 2002;277(14):12158-63.

6. Kubo Y, Baldwin TJ, Jan YN, Jan LY. Primary structure and functional expression of a mouse inward rectifier potassium channel. Nature. 1993;362(6416):127-33.

7. Hibino H, Inanobe A, Furutani K, Murakami S, Findlay I, Kurachi Y. Inwardly rectifying potassium channels: their structure, function, and physiological roles. Physiol Rev. 2010;90(1):291-366.

8. Lopatin AN, Nichols CG. Inward rectifiers in the heart: an update on I (K1). J Mol Cell Cardiol. 2001;33(4):625-38.

9. Pancrazio JJ, Tabbara IA, Kim YI. Voltage-activated K+ conductance and cel proliferation in small-cell lung cancer. Anticancer Res. 1993;13(4):1231-4.

10. Andersen ED, Krasilnikoff PA, Overvad H. Intermittent muscular weakness, extrasystoles, and multiple developmental anomalies. A new syndrome. Acta Paediatr Scand. 1971;60(5):559-64.

11. Plaster NM, Tawil R, Tristani-Firouzi M, Canun S, Bendahhou S, Tsunoda A, et al. Mutations in Kir2.1 cause the developmental and episodic electrical phenotypes of Andersen's syndrome. Cell. 2001;105(4):511-9.

12. Preisig-Muller R, Schlichthorl G, Goerge T, Heinen S, Bruggemann A, Rajan S, et al. Heteromerization of Kir2.x potassium channels contributes to the phenotype of Andersen's syndrome. Proc Natl Acad Sci U S A. 2002;99(11):7774-9.

13. Sansone V, Griggs RC, Meola G, Ptacek LJ, Barohn R, lannaccone S, et al. Andersen's syndrome: a distinct periodic paralysis. Ann Neurol. 1997:42(3):305-12

14. Tawil R, Ptacek LJ, Pavlakis SG, DeVivo DC, Penn AS, Ozdemir C, et al. Andersen's syndrome: potassium-sensitive periodic paralysis, ventricular ectopy, and dysmorphic features. Ann Neurol. 1994;35(3):326-30.
15. Xia M, Jin $Q$, Bendahhou S, He Y, Larroque MM, Chen $Y$, et al. A Kir2.1 gainof-function mutation underlies familial atrial fibrillation. Biochem Biophys Res Commun. 2005;332(4):1012-9.

16. Jirsch J, Deeley RG, Cole SP, Stewart AJ, Fedida D. Inwardly rectifying K+ channels and volume-regulated anion channels in multidrug-resistant small cell lung cancer cells. Cancer Res. 1993;53(18):4156-60.

17. Sakai H, Shimizu T, Hori K, Ikari A, Asano S, Takeguchi N. Molecular and pharmacological properties of inwardly rectifying $\mathrm{K}+$ channels of human lung cancer cells. Eur J Pharmacol. 2002;435(2-3):125-33.

18. Bartel DP. MicroRNAs: genomics, biogenesis, mechanism, and function. Cell. 2004;116(2):281-97.

19. Ambros $V$. The functions of animal microRNAs. Nature. 2004;431(7006):350-5.

20. He L, Hannon GJ. MicroRNAs: small RNAs with a big role in gene regulation. Nat Rev Genet. 2004;5(7):522-31.

21. Goldoni D, Yarham JM, McGahon MK, O'Connor A, Guduric-Fuchs J, Edgar K, et al. A novel dual-fluorescence strategy for functionally validating microRNA targets in $3^{\prime}$ untranslated regions: regulation of the inward rectifier potassium channel K (ir) 2.1 by miR-212. Biochem J. 2012;448(1):103-13.

22. Guo L, Liu Y, Bai Y, Sun Y, Xiao F, Guo Y. Gene expression profiling of drug-resistant small cell lung cancer cells by combining microRNA and cDNA expression analysis. Eur J Cancer. 2010;46(9):1692-702.

23. Huang JY, Cui SY, Chen YT, Song HZ, Huang GC, Feng B, et al. MicroRNA650 was a prognostic factor in human lung adenocarcinoma and confers the docetaxel chemoresistance of lung adenocarcinoma cells via regulating BCl-2/Bax expression. PLoS One. 2013;8(8):e72615.

24. Wang Y, Gu X, Li Z, Xiang J, Jiang J. Chen Z: microRNA expression profiling in multidrug resistance of the 5Fuinduced SGC7901 human gastric cancer cell line. Mol Med Rep. 2013;7(5):1506-10.

25. Yang H, Kong W, He L, Zhao JJ, O'Donnell JD, Wang J, et al. MicroRNA expression profiling in human ovarian cancer: miR-214 induces cell survival and cisplatin resistance by targeting PTEN. Cancer Res. 2008;68(2):425-33.

26. Li P, Ma L, Zhang Y, Ji F, Jin F. MicroRNA-137 down-regulates KIT and inhibits small cell lung cancer cell proliferation. Biomed Pharmacother. 2014;68(1):7-12.

27. Garofalo M. Croce CM: microRNAs: Master regulators as potential therapeutics in cancer. Annu Rev Pharmacol Toxicol. 2011;51:25-43.

28. Fakler B, Brandle U, Glowatzki E, Zenner HP, Ruppersberg JP. Kir2.1 inward rectifier $\mathrm{K}+$ channels are regulated independently by protein kinases and ATP hydrolysis. Neuron. 1994;13(6):1413-20.

29. Pancrazio JJ, Viglione MP, Tabbara IA, Kim YI. Voltage-dependent ion channels in small-cell lung cancer cells. Cancer Res. 1989;49(21):5901-6.

30. Ji L, Li H, Gao P, Shang G, Zhang DD, Zhang N, et al. Nrf2 pathway regulates multidrug-resistance-associated protein 1 in small cell lung cancer. PLoS One. 2013;8(5):e63404.

31. Lam HD, Lemay AM, Kelly J, Hill CE. Loss of Kv and MaxiK currents associated with increased MRP1 expression in small cell lung carcinoma. J Cell Physiol. 2006;209(2):535-41.

32. Enyeart JJ, Xu L, Danthi S, Enyeart JA. An ACTH- and ATP-regulated background $\mathrm{K}+$ channel in adrenocortical cells is TREK-1. J Biol Chem. 2002;277(51):49186-99.

33. Alesutan I, Munoz C, Sopjani M, Dermaku-Sopjani M, Michael D, Fraser S, et al. Inhibition of Kir2.1 (KCNJ2) by the AMP-activated protein kinase. Biochem Biophys Res Commun. 2011;408(4):505-10.

34. Scherer D, Kiesecker C, Kulzer M, Gunth M, Scholz EP, Kathofer S, et al. Activation of inwardly rectifying Kir2.x potassium channels by beta 3-adrenoceptors is mediated via different signaling pathways with a predominant role of PKC for Kir2.1 and of PKA for Kir2.2. Naunyn Schmiedebergs Arch Pharmacol. 2007;375(5):311-22.

35. Wischmeyer E, Doring F, Karschin A. Acute suppression of inwardly rectifying Kir2.1 channels by direct tyrosine kinase phosphorylation. J Biol Chem. 1998;273(51):34063-8.

36. Chen Z, Ma T, Huang C, Zhang L, Lv X, Xu T, et al. MiR-27a modulates the MDR1/P-glycoprotein expression by inhibiting FZD7/beta-catenin pathway in hepatocellular carcinoma cells. Cell Signal. 2013;25(12):2693-701.

37. Fang Y, Xue JL, Shen Q, Chen J, Tian L. MicroRNA-7 inhibits tumor growth and metastasis by targeting the phosphoinositide 3-kinase/Akt pathway in hepatocellular carcinoma. Hepatology. 2012;55(6):1852-62.

38. Kefas B, Godlewski J, Comeau L, Li Y, Abounader R, Hawkinson M, et al. microRNA-7 inhibits the epidermal growth factor receptor and the Akt pathway and is down-regulated in glioblastoma. Cancer Res. 2008;68(10):3566-72 
39. Zhao X, Dou W, He L, Liang S, Tie J, Liu C, et al. MicroRNA-7 functions as an anti-metastatic microRNA in gastric cancer by targeting insulin-like growth factor-1 receptor. Oncogene. 2013;32(11):1363-72.

40. Zhang N, Li X, Wu CW, Dong Y, Cai M, Mok MT, et al. microRNA-7 is a novel inhibitor of YY1 contributing to colorectal tumorigenesis. Oncogene. 2013;32(42):5078-88.

41. Livak KJ, Schmittgen TD. Analysis of relative gene expression data using real-time quantitative PCR and the 2 (-Delta Delta C (T)) Method. Methods. 2001;25(4):402-8

42. Xiao F, Bai Y, Chen Z, Li Y, Luo L, Huang J, et al. Downregulation of HOXA1 gene affects small cell lung cancer cell survival and chemoresistance under the regulation of miR-100. Eur J Cancer. 2014;50(8):1541-54

\section{Submit your next manuscript to BioMed Central and take full advantage of:}

- Convenient online submission

- Thorough peer review

- No space constraints or color figure charges

- Immediate publication on acceptance

- Inclusion in PubMed, CAS, Scopus and Google Scholar

- Research which is freely available for redistribution 